Dynamics of ionization wave splitting and merging of atmospheric-pressure plasmas in branched dielectric tubes and channels

This article has been downloaded from IOPscience. Please scroll down to see the full text article.

2012 J. Phys. D: Appl. Phys. 45275201

(http://iopscience.iop.org/0022-3727/45/27/275201)

View the table of contents for this issue, or go to the journal homepage for more

Download details:

IP Address: 141.211.173.82

The article was downloaded on 26/06/2013 at 15:02

Please note that terms and conditions apply. 


\title{
Dynamics of ionization wave splitting and merging of atmospheric-pressure plasmas in branched dielectric tubes and channels
}

\author{
Zhongmin Xiong $^{1}$, Eric Robert ${ }^{2}$, Vanessa Sarron ${ }^{2}$, Jean-Michel Pouvesle ${ }^{2}$ \\ and Mark J Kushner ${ }^{1}$ \\ ${ }^{1}$ Electrical Engineering and Computer Science Dept., University of Michigan, Ann Arbor, \\ MI 48109, USA \\ ${ }^{2}$ GREMI, UMR7344, CNRS Université d'Orléans, 45067 Orléans Cedex 2, France \\ E-mail: zxiong@umich.edu,eric.robert@univ-orleans.fr, vanessa.sarron@univ-orleans.fr, \\ jean-michel.pouvesle@univ-orleans.fr and mjkush@umich.edu
}

Received 27 February 2012, in final form 21 April 2012

Published 15 June 2012

Online at stacks.iop.org/JPhysD/45/275201

\begin{abstract}
Atmospheric-pressure fast ionization waves (FIWs) generated by nanosecond, high voltage pulses are able to propagate long distances through small diameter dielectric tubes or channels, and so deliver UV fluxes, electric fields, charged and excited species to remote locations. In this paper, the dynamics of FIW splitting and merging in a branched dielectric channel are numerically investigated using a two-dimensional plasma hydrodynamics model with radiation transport, and the results are compared with experiments. The channel consists of a straight inlet section branching $90^{\circ}$ into a circular loop which terminates to form a second straight outlet section aligned with the inlet section. The plasma is sustained in neon gas with a trace amount of xenon at atmospheric pressure. The FIW generated at the inlet approaches the first branch point with speeds of $\approx 10^{8} \mathrm{~cm} \mathrm{~s}^{-1}$, and produces a streamer at the inlet-loop junction. The induced streamer then splits into two FIW fronts, each propagating in opposite directions through half of the loop channel. The FIWs slow as they traverse the circular sections due to a shorting of the electric field by the other FIW. Approaching the loop-outlet junction, the two FIW fronts nearly come to a halt, induce another streamer which goes through further splitting and finally develops into a new FIW front. The new FIW increases in speed and plasma density propagating in the straight outlet channel. The electrical structure of the FIWs and the induced streamers during the splitting and merging processes are discussed with an emphasis on their mutual influence and their interaction with the channel wall. The FIW propagation pattern is in good agreement with experimental observations. Based on numerical and experimental investigations, a model for the splitting and merging FIWs in the branched loop channel is proposed.
\end{abstract}

(Some figures may appear in colour only in the online journal)

\section{Introduction}

Fast ionization waves (FIWs) generated by nanosecond, highvoltage pulses in atmospheric-pressure plasmas are capable of producing significant ionization, intense electric fields, UV fluxes, and high concentrations of charged and neutral excited species at high pressure [1-3]. Atmospheric-pressure
FIWs are of interest for many applications ranging from plasma-assisted combustion and high-speed aeronautical flow control, to the preionization of electric discharge excited gas lasers $[4,5]$. In recent years, many cold, atmospheric-pressure plasma devices excited by radio frequency (rf) or repetitive waveforms, such as the plasma needle and plasma jets [6-8], have been developed in the context of plasma medicine-the 
interaction between low-temperature plasmas and living cells and tissues. Although these repetitively excited plasma jets typically appear as a luminous continuum, high-speed imaging has shown that they are composed of a series of fast travelling plasmas bullets or ionization wave fronts $[8,9]$. Confined within narrow dielectric tubes, rigid or flexible, the ionization wave front is capable of travelling a distance of up to ten to hundreds of centimetres at speeds of $10^{7}-10^{8} \mathrm{~cm} \mathrm{~s}^{-1}$, and exit the tube as a plasma jet [10-12].

The ability of FIWs to generate and deliver plasma species and the associated electric fields and photon fluxes through small diameter tubes to remote locations opens up new potential applications. These applications include thinfilm deposition on the inner surface of the tubes, plasma sterilization of catheters, and treating internal organs and body cavities by delivering cold plasmas through endoscopic devices [13-15]. The dynamics of plasma bullets or FIW fronts during their propagation through capillaries, particularly at the junctions or splitting of capillary tubes, ultimately determine the fluxes of radicals and ions delivered at the end of the capillary. In principle, the properties of such junctions can be used as control points where certain measures (e.g., radius of curvature, dielectric constant, radius of channel) may be used to regulate the FIWs, such as redirecting to different paths or adjusting their strengths. Recent experiments have shown that plasma jets, for which the new moniker of pulsed atmospheric-pressure plasma streams was suggested, can be delivered by branched capillary tubes with different junctions to perform in vivo treatment of tumours in the internal organs of mice $[16,17]$.

FIW in shielded tubes or channels [1-3, 10], sometimes termed as e-solitons [2], have been previously investigated but the dynamics of branching of FIW and the interaction of colliding FIW fronts is less well understood. In this paper, we report on numerical and experimental investigations of atmospheric-pressure FIW fronts propagating through a thin branched loop channel filled with neon, focusing on the splitting and merging processes at the channel junctions. By comparison with experiments, the goal is to obtain an improved understanding of the splitting and merging of FIW, their mutual influence and their interaction with the bounding surfaces. We found that the FIWs propagate in a wall-hugging mode inside the channel, and can shift from one side to the other on curved sections. The splitting and merging of FIWs at channel junctions may be facilitated by an intermediate streamer discharge that is induced by the approaching FIWs. At the merging junction, the induced streamer goes through further splitting and finally develops into a new FIW front with enhanced speed and intensity that continues to propagate in the outlet channel.

Brief descriptions of the two-dimensional (2D) modelling platform, experimental diagnostics and the discharge configuration used in this investigation are in section 2 . Numerical results for the splitting and merging of FIWs are presented and compared with experimental observations in section 3. Based on the numerical and experimental studies, a model of the FIW propagation in the branched dielectric loop channels is proposed in section 4. Our discussion and concluding remarks are in section 5 .

\section{Description of the model and experiment}

\subsection{Description of the model}

The 2D simulations of FIWs in channels sustained in neon were performed using non-PDPSIM, a plasma hydrodynamics model with radiation transport, and is described in detail in [18]. We briefly summarize the model here. In nonPDPSIM, continuity equations for charged and neutral species, and Poisson's equation for electric potential are integrated coincidently in time with the electron energy equation using transport coefficients obtained from stationary solutions of the Boltzmann equation. The use of the electron energy equation allows for non-equilibrium between the local electric field and electron transport coefficients. The specific equations solved are

$$
\begin{gathered}
-\nabla \cdot \varepsilon \nabla \Phi=\sum_{j} n_{j} q_{j}+\rho_{\mathrm{s}}, \\
\frac{\partial N_{i}}{\partial t}=-\nabla \cdot \vec{\phi}_{i}+S_{i}, \\
\frac{\partial \rho_{\mathrm{s}}}{\partial t}=\sum_{i}-\nabla \cdot\left(q_{i} \vec{\phi}_{i}\left(1+\gamma_{i}\right)\right)+\nabla \cdot(\sigma \nabla \Phi),
\end{gathered}
$$

where $\varepsilon, \Phi, \rho_{\mathrm{s}}, N, \phi, \gamma, \sigma, S$ and $q$ are the permittivity, electric potential, surface charge density, charged species number density, species flux, secondary electron emission coefficient, conductivity of the solid materials, source terms and elementary charge. The source term $S_{i}$ contains the production and loss of species $i$ due to electron-impact ionization and excitation, heavy particle reactions, photoionization, secondary emission and surface reactions. The charged particle fluxes $\phi_{i}$ are approximated with the ScharfetterGummel technique and equations (1)-(3) are simultaneously solved in each Newton iteration step. The charge density $\rho_{\mathrm{s}}$ is computed both in the volume and on surfaces. The charge density on surfaces is then included in the solution of the electric potential in the same way that charge density is included in the gas phase. That is, we do not explicitly employ a boundary condition on electric field on surfaces. For sufficiently fine meshing, our technique provides the same electric configuration as imposing a boundary condition on electric field. The spatial discretization used in non-PDPSIM is based on a finite volume method on an unstructured mesh, and is formulated to be $100 \%$ conservative.

Once the charged particle densities are updated, the electron energy equation is integrated for average energy $\varepsilon$

$$
\begin{gathered}
\frac{\partial}{\partial t}\left(n_{\mathrm{e}} \varepsilon\right)=q \vec{\phi}_{\mathrm{e}} \cdot \vec{E}-n_{\mathrm{e}} \sum_{i} N_{i} k_{i} \Delta \varepsilon_{i} \\
-\nabla \cdot\left(\frac{5}{2} \varepsilon \vec{\phi}_{\mathrm{e}}-\lambda \nabla T_{\mathrm{e}}\right),
\end{gathered}
$$

where $n_{\mathrm{e}}$ is the electron density, $E=-\nabla \Phi$ is the electric field, $k_{i}$ is the rate coefficient for collision process $i$ with species having density $N_{i}$ and energy loss $\Delta \varepsilon_{i}, \lambda$ is the electron thermal conductivity and $\vec{\phi}_{\mathrm{e}}$ is the electron flux and $T_{\mathrm{e}}$ is electron temperature defined as $(2 \varepsilon / 3)$. For elastic collisions with an atom or molecule having energy $M_{i}$, the energy loss is $\Delta \varepsilon_{i}=\left(2 m_{\mathrm{e}} / M_{i}\right) \varepsilon$, for electron mass $m_{\mathrm{e}}$. 
During the short duration of the streamer discharge, advective motion of neutral species by temperature or pressure gradients is not important. The densities of neutral species are given by their continuity equations with only diffusion for transport. These continuity equations for neutral species are solved in a time-slicing manner with the charged particle continuity equations,

$$
\frac{\partial N_{i}}{\partial t}=-\nabla \cdot\left(-D_{i} N_{\mathrm{T}} \nabla\left(\frac{N_{i}}{N_{\mathrm{T}}}\right)\right)+S_{i},
$$

where the $N_{i}$ is the density of neutral species $i, N_{\mathrm{T}}$ is the total gas density, $D_{i}$ is the diffusion coefficient and $S_{i}$ is the source or sink due to volumetric or surface processes.

Photon transport in the plasma is accounted for using a propagator method. The photoionization source for species $m$ at location $\vec{r}_{i}$ due to the emission of photons at location $\vec{r}_{j}$ by species $k$ is

$$
\begin{gathered}
S_{m}\left(\vec{r}_{i}\right)=\sigma_{k m}^{I} N_{m}\left(\vec{r}_{i}\right) A_{k} \int N_{k}\left(\vec{r}_{j}^{\prime}\right) G_{k}\left(\vec{r}_{j}^{\prime}, \vec{r}_{i}\right) \mathrm{d}^{3} \vec{r}_{j}^{\prime}, \\
G_{k}\left(\vec{r}_{j}^{\prime}, \vec{r}_{i}\right)=\frac{\exp \left(-\int_{\vec{r}_{j}^{\prime}}^{\vec{r}_{i n}^{\prime}} \sum_{l} \sigma_{l k} N_{l}\left(\vec{r}_{n}^{\prime}\right) \mathrm{d} \vec{r}_{n}^{\prime}\right)}{4 \pi\left|\vec{r}_{i}^{\prime}-\vec{r}_{j}\right|^{2}},
\end{gathered}
$$

where $N_{k}$ is the density of the radiating species having the Einstein coefficient $A_{k}, \sigma_{k m}^{I}$ is the photoionization cross section for species $m$ by photons emitted by species $k$, and $\sigma_{l k}$ is the total absorption cross section for photon $k$ by species $l$. $G_{k}\left(\vec{r}_{j}^{\prime}, \vec{r}_{i}\right)$ is Green's function for the survival of the photons emitted at the location $\vec{r}_{j}$ to reach location $\vec{r}_{i}$, and also accounts for view angles and obscurations.

The gas mixture is $\mathrm{Ne} / \mathrm{Xe}$ at atmospheric pressure and $300 \mathrm{~K}$, and the reaction mechanism is similar to that discussed in [19] and is listed in table 1. The Xe model consists of ground state $\mathrm{Xe}$, singly charged ion $\mathrm{Xe}^{+}, \mathrm{Xe}^{*}$ (6s states), $\mathrm{Xe}^{* *}$ (6s' states), a composite excited state $\mathrm{Xe}^{* * *}$ (nominally $\mathrm{Xe}(6 \mathrm{p}$, $5 \mathrm{~d})$ ), the dimer excited state $\mathrm{Xe}_{2}^{*}$, and the dimer ion $\mathrm{Xe}_{2}^{+}$. The radiative $6 \mathrm{~s}$ state is lumped into $\mathrm{Xe}^{*}$ and the radiative $6 \mathrm{~s}^{\prime}$ state is lumped into $\mathrm{Xe}^{* *}$. The $\mathrm{Ne}$ model consists of $\mathrm{Ne}$ (ground state), $\mathrm{Ne}^{*}$, a composite excited state nominally $\mathrm{Ne}(3 \mathrm{~s}), \mathrm{Ne}^{+}$, $\mathrm{Ne}_{2}^{*}$ and $\mathrm{Ne}_{2}^{+}$.

The purpose of adding a small admixture of Xe to $\mathrm{Ne}$ is two fold. First, doing so removes the ambiguity on the source of photoionization. In the non-pristine feedstock gases, photoionization of trace impurities likely occurs by photons from excited states of neon, and the $\mathrm{Ne}_{2}^{*}$ dimer in particular. In our reaction mechanism, photoionization of Xe by $85 \mathrm{~nm}$ radiation from $\mathrm{Ne}_{2}^{*}$ emission is included with a cross section of $10^{-16} \mathrm{~cm}^{2}$. In the region ahead of the FIW front, the photoionization generates seed electrons, in addition to those generated by electron drift, although the latter is dominant for the negative FIW in this study. Second, because of its lower ionization potential, a small amount of Xe can enhance the electron-impact ionization considerably, and thus affect the propagation distance and speed of the FIW. The fraction of Xe in this study was varied from $0 \%$ to $1 \%$, with the base case having an impurity level of $0.1 \%$.
We included secondary electron emission from plasma bounding surfaces due to ion bombardment. The exact value of the secondary electron emission coefficient $\gamma$ is largely unknown and in the literature it typically varies between 0.01 and 0.20 [20]. In this study we used $\gamma=0.15$. The surface secondary electrons are not expected to play an overly significant role here due to the short FIW time scale during which the ions do not have significant fluxes to the surfaces. The secondary photoelectron emission from these surfaces was neglected for simplicity.

Due to the 2D nature of our model, the dielectric cylindrical tubes used in the experiments were approximated by channels in the simulations. Similar to the apparatus discussed in [21], the branched dielectric channel consists of a straight inlet section that contains the initiating electrodes which intersects at $90^{\circ}$ with a circular section. (See figure 1.) A second straight outlet section is aligned with the inlet section on the axis of symmetry. The full computational domain (not shown in figure 1) is a $23 \times 20 \mathrm{~cm}^{2}$ rectangle filled with ambient air with all of the boundaries grounded. The lengths of the inlet and outlet sections are 2.5 and $5 \mathrm{~cm}$, and the outer diameter of the loop section is $5 \mathrm{~cm}$. The width of the channel is $4 \mathrm{~mm}$ and the thickness of the glass wall $\left(\varepsilon_{r}=4\right)$ is $1 \mathrm{~mm}$. The total number of the nodes in the numerical mesh is about 13000 with 9100 points in the plasma region. The mesh size inside the dielectric channel is typically about $100 \mu \mathrm{m}$. A simulation with finer mesh size $(\approx 40 \mu \mathrm{m})$ was also carried out and the results show no significant differences from those with the coarser mesh. The total simulation time is $1 \mu \mathrm{s}$ which takes about $48 \mathrm{~h}$ on a workstation having 8 -core $3.5 \mathrm{GHz}$ xeon processors.

The FIW is initiated at the inlet between a pair of grounded electrodes outside the channel and a needle-like, powered electrode inside the channel which is supplied with a $-25 \mathrm{kV}$ pulse with $25 \mathrm{~ns}$ rise time. The voltage is held constant afterwards. The length and width of the needle is 0.3 and $0.02 \mathrm{~cm}$ and the radius of curvature at the needle tip is $0.01 \mathrm{~cm}$. The initial electron density inside the channel is zero except for a small charge neutral electron-ion cloud having radius of $250 \mu \mathrm{m}$ with $[\mathrm{e}] \approx 10^{10} \mathrm{~cm}^{-3}$ near the tip of the powered electrode. No excited states are initialized. This cloud was used so that there would be an unambiguous starting time for the discharge. The spatial extent and magnitude of this initial cloud were extensively parametrized to ensure that our choice does not affect the final results.

In the discussion that follows the terms FIW and streamer are used to describe the plasma in different parts of the channels. It is true that streamers are or can be FIW. However, in this context, streamer is used to describe FIWs that are launched mostly at junctions of the tube in the volume of the channel and which are not terribly affected by the walls.

\subsection{Experimental setup}

A few modifications have been implemented in the experimental setup first reported in [21] to image and characterize neon atmospheric-pressure plasma generation, splitting and mixing in branched tubes. The goal was to match 
Table 1. Ne/Xe reaction mechanism.

\begin{tabular}{|c|c|c|}
\hline Reaction & Rate coefficient & Reference \\
\hline \multicolumn{3}{|c|}{ Electron-impact excitation and de-excitation } \\
\hline $\mathrm{e}+\mathrm{Xe} \rightarrow \mathrm{Xe}+\mathrm{e}$ & $\Delta \varepsilon=\varepsilon\left(2 m_{\mathrm{e}} / M\right)^{\mathrm{a}}$ & \\
\hline $\mathrm{e}+\mathrm{Xe} \rightarrow \mathrm{Xe}^{*}+\mathrm{e}$ & $\Delta \varepsilon=8.4 \mathrm{eV}^{\mathrm{a}}$ & [23] \\
\hline $\mathrm{e}+\mathrm{Xe} \rightarrow \mathrm{Xe}^{* *}+\mathrm{e}$ & $\Delta \varepsilon=9.5 \mathrm{eV}^{\mathrm{a}}$ & {$[24]$} \\
\hline $\mathrm{e}+\mathrm{Xe} \rightarrow \mathrm{Xe}^{* * *}+\mathrm{e}$ & $\Delta \varepsilon=9.8 \mathrm{eV}^{\mathrm{a}}$ & {$[25]$} \\
\hline $\mathrm{e}+\mathrm{Xe}^{*} \rightarrow \mathrm{Xe}+\mathrm{e}$ & $\Delta \varepsilon=-8.4 \mathrm{eV}^{\mathrm{a}}$ & [26] \\
\hline $\mathrm{e}+\mathrm{Xe}^{* *} \rightarrow \mathrm{Xe}+\mathrm{e}$ & $\Delta \varepsilon=-9.5 \mathrm{eV}^{\mathrm{a}}$ & {$[24]^{\mathrm{b}}$} \\
\hline $\mathrm{e}+\mathrm{Xe}^{* * *} \rightarrow \mathrm{Xe}+\mathrm{e}$ & $\Delta \varepsilon=-9.8 \mathrm{eV}^{\mathrm{a}}$ & {$[25]^{\mathrm{b}}$} \\
\hline $\mathrm{e}+\mathrm{Xe}^{*} \rightarrow \mathrm{Xe}^{* * *}+\mathrm{e}$ & $\Delta \varepsilon=1.4 \mathrm{eV}^{\mathrm{a}}$ & {$[26]$} \\
\hline $\mathrm{e}+\mathrm{Xe}^{* * *} \rightarrow \mathrm{Xe}^{*}+\mathrm{e}$ & $\Delta \varepsilon=-1.4 \mathrm{eV}^{\mathrm{a}}$ & {$[26]^{\mathrm{b}}$} \\
\hline $\mathrm{e}+\mathrm{Xe}^{* *} \rightarrow \mathrm{Xe}^{* * *}+\mathrm{e}$ & $5.5 \times 10^{-7} T_{\mathrm{e}}^{0.79} \exp \left(-2.0 / T_{\mathrm{e}}\right), \Delta \varepsilon=0.3 \mathrm{eV}$ & {$[27]$} \\
\hline $\mathrm{e}+\mathrm{Ne} \rightarrow \mathrm{Me}+\mathrm{e}$ & $\Delta \varepsilon=\varepsilon\left(2 m_{\mathrm{e}} / M\right)^{\mathrm{a}}$ & \\
\hline $\mathrm{e}+\mathrm{Ne} \rightarrow \mathrm{Ne}^{*}+\mathrm{e}$ & $\Delta \varepsilon=20.6 \mathrm{eV}^{\mathrm{a}}$ & [28] \\
\hline $\mathrm{e}+\mathrm{Ne}^{*} \rightarrow \mathrm{Ne}+\mathrm{e}$ & $\Delta \varepsilon=-20.6 \mathrm{eV}^{\mathrm{a}}$ & {$[28]^{\mathrm{b}}$} \\
\hline \multicolumn{3}{|c|}{ Electron-impact ionization and recombination } \\
\hline $\mathrm{e}+\mathrm{Xe} \rightarrow \mathrm{Xe}^{+}+\mathrm{e}+\mathrm{e}$ & $\Delta \varepsilon=12.1 \mathrm{eV}^{\mathrm{a}}$ & [29] \\
\hline $\mathrm{e}+\mathrm{Xe}^{*} \rightarrow \mathrm{Xe}^{+}+\mathrm{e}+\mathrm{e}$ & $\Delta \varepsilon=3.7 \mathrm{eV}^{\mathrm{a}}$ & {$[26]$} \\
\hline $\mathrm{e}+\mathrm{Xe}^{* *} \rightarrow \mathrm{Xe}^{+}+\mathrm{e}+\mathrm{e}$ & $\Delta \varepsilon=2.3 \mathrm{eV}^{\mathrm{a}}$ & {$[26]$} \\
\hline $\mathrm{e}+\mathrm{Xe}^{* * *} \rightarrow \mathrm{Xe}^{+}+\mathrm{e}+\mathrm{e}$ & $1.56 \times 10^{-7} T_{\mathrm{e}}^{0.71} \exp \left(-2.63 / T_{\mathrm{e}}\right), \Delta \varepsilon=2.6 \mathrm{eV}$ & [27] \\
\hline $\mathrm{e}+\mathrm{Xe}_{2}^{*} \rightarrow \mathrm{Xe}_{2}^{+}+\mathrm{e}+\mathrm{e}$ & $9.75 \times 10^{-8} T_{\mathrm{e}}^{0.71} \exp \left(-3.4 / T_{\mathrm{e}}\right), \Delta \varepsilon=3.3 \mathrm{eV}$ & [27] \\
\hline $\mathrm{e}+\mathrm{Xe}_{2}^{+} \rightarrow \mathrm{Xe}^{*}+\mathrm{Xe}$ & $0.37 \times 10^{-7} T_{\mathrm{e}}^{-0.5}$ & {$[30,31]$} \\
\hline $\mathrm{e}+\mathrm{Xe}_{2}^{+} \rightarrow \mathrm{Xe}^{* *}+\mathrm{Xe}$ & $0.37 \times 10^{-7} T_{\mathrm{e}}^{-0.5}$ & {$[30,31]$} \\
\hline $\mathrm{e}+\mathrm{Xe}_{2}^{+} \rightarrow \mathrm{Xe}^{* * *}+\mathrm{Xe}$ & $3.33 \times 10^{-7} T_{\mathrm{e}}^{-0.5}$ & {$[30,31]$} \\
\hline $\mathrm{e}+\mathrm{Ne} \rightarrow \mathrm{Ne}^{+}+\mathrm{e}+\mathrm{e}$ & $\Delta \varepsilon=21.6 \mathrm{eV}^{\mathrm{a}}$ & {$[29]$} \\
\hline $\mathrm{e}+\mathrm{Ne}^{*} \rightarrow \mathrm{Ne}^{+}+\mathrm{e}+\mathrm{e}$ & $\Delta \varepsilon=1.0 \mathrm{eV}^{\mathrm{a}}$ & [32] \\
\hline $\mathrm{e}+\mathrm{Ne}_{2}^{*} \rightarrow \mathrm{Ne}_{2}^{+}+\mathrm{e}+\mathrm{e}$ & $4.93 \times 10^{-6} T_{\mathrm{e}}^{0.68} \exp \left(-6.45 / T_{\mathrm{e}}\right), \Delta \varepsilon=5.0 \mathrm{eV}$ & [27] \\
\hline $\mathrm{e}+\mathrm{Ne}_{2}^{+} \rightarrow \mathrm{Ne}^{*}+\mathrm{Ne}$ & $3.72 \times 10^{-8} T_{\mathrm{e}}^{-0.43}$ & {$[30,31]$} \\
\hline \multicolumn{3}{|c|}{ Two-body heavy particle collisions } \\
\hline $\mathrm{Xe}^{*}+\mathrm{Xe}^{*} \rightarrow \mathrm{Xe}^{+}+\mathrm{Xe}+\mathrm{e}$ & $5.0 \times 10^{-10}$ & [33] \\
\hline $\mathrm{Xe}^{* *}+\mathrm{Xe}^{* *} \rightarrow \mathrm{Xe}^{+}+\mathrm{Xe}+\mathrm{e}$ & $5.0 \times 10^{-10}$ & {$[33]^{\mathrm{c}}$} \\
\hline $\mathrm{Xe}^{* * *}+\mathrm{Xe}^{* * *} \rightarrow \mathrm{Xe}^{+}+\mathrm{Xe}+\mathrm{e}$ & $5.0 \times 10^{-10}$ & {$[33]^{\mathrm{c}}$} \\
\hline $\mathrm{Ne}^{*}+\mathrm{Ne}^{*} \rightarrow \mathrm{Ne}^{+}+\mathrm{Ne}+\mathrm{e}$ & $1.0 \times 10^{-11}$ & {$[27]$} \\
\hline $\mathrm{Ne}^{+}+\mathrm{Xe} \rightarrow \mathrm{Ne}+\mathrm{Xe}^{+}$ & $1.0 \times 10^{-11}$ & [27] \\
\hline $\mathrm{Ne}^{*}+\mathrm{Xe} \rightarrow \mathrm{Xe}^{+}+\mathrm{Ne}+\mathrm{e}$ & $1.0 \times 10^{-10}$ & [27] \\
\hline $\mathrm{Ne}_{2}^{+}+\mathrm{Xe} \rightarrow \mathrm{Xe}^{+}+\mathrm{Ne}+\mathrm{Ne}$ & $1.0 \times 10^{-10}$ & {$[27]$} \\
\hline $\mathrm{Ne}_{2}^{*}+\mathrm{Xe} \rightarrow \mathrm{Xe}^{+}+\mathrm{Ne}+\mathrm{Ne}+\mathrm{e}$ & $7.5 \times 10^{-11}$ & {$[27]$} \\
\hline \multicolumn{3}{|c|}{ Three-body heavy particle collisions } \\
\hline $\mathrm{Xe}^{*}+\mathrm{Xe}+\mathrm{Xe} \rightarrow \mathrm{Xe}_{2}^{*}+\mathrm{Xe}$ & $1.0 \times 10^{-32} \mathrm{~cm}^{6} \mathrm{~s}^{-1}$ & [34] \\
\hline $\mathrm{Xe}^{*}+\mathrm{Xe}+\mathrm{Ne} \rightarrow \mathrm{Xe}_{2}^{*}+\mathrm{Ne}$ & $1.0 \times 10^{-32} \mathrm{~cm}^{6} \mathrm{~s}^{-1}$ & [35] \\
\hline $\mathrm{Xe}^{* *}+\mathrm{Xe}+\mathrm{Xe} \rightarrow \mathrm{Xe}_{2}^{*}+\mathrm{Xe}$ & $2.0 \times 10^{-31} \mathrm{~cm}^{6} \mathrm{~s}^{-1}$ & [34] \\
\hline $\mathrm{Xe}^{* *}+\mathrm{Xe}+\mathrm{Ne} \rightarrow \mathrm{Xe}_{2}^{*}+\mathrm{Ne}$ & $2.0 \times 10^{-31} \mathrm{~cm}^{6} \mathrm{~s}^{-1}$ & {$[35]^{\mathrm{c}}$} \\
\hline $\mathrm{Xe}^{* * *}+\mathrm{Xe}+\mathrm{Xe} \rightarrow \mathrm{Xe}_{2}^{*}+\mathrm{Xe}$ & $5.0 \times 10^{-32} \mathrm{~cm}^{6} \mathrm{~s}^{-1}$ & {$[34]$} \\
\hline $\mathrm{Xe}^{* * *}+\mathrm{Xe}+\mathrm{Ne} \rightarrow \mathrm{Xe}_{2}^{*}+\mathrm{Ne}$ & $5.0 \times 10^{-32} \mathrm{~cm}^{6} \mathrm{~s}^{-1}$ & {$[35]^{\mathrm{c}}$} \\
\hline $\mathrm{Xe}^{+}+\mathrm{Xe}+\mathrm{Xe} \rightarrow \mathrm{Xe}_{2}^{+}+\mathrm{Xe}$ & $1.5 \times 10^{-31} \mathrm{~cm}^{6} \mathrm{~s}^{-1}$ & [35] \\
\hline $\mathrm{Xe}^{+}+\mathrm{Xe}+\mathrm{Ne} \rightarrow \mathrm{Xe}_{2}^{+}+\mathrm{Ne}$ & $1.5 \times 10^{-31} \mathrm{~cm}^{6} \mathrm{~s}^{-1}$ & {$[35]$} \\
\hline $\mathrm{Ne}^{*}+\mathrm{Ne}+\mathrm{Xe} \rightarrow \mathrm{Ne}_{2}^{*}+\mathrm{Xe}$ & $8.0 \times 10^{-34} \mathrm{~cm}^{6} \mathrm{~s}^{-1}$ & [27] \\
\hline $\mathrm{Ne}^{*}+\mathrm{Ne}+\mathrm{Ne} \rightarrow \mathrm{Ne}_{2}^{*}+\mathrm{Ne}$ & $8.0 \times 10^{-34} \mathrm{~cm}^{6} \mathrm{~s}^{-1}$ & {$[35]$} \\
\hline $\mathrm{Ne}^{+}+\mathrm{Ne}+\mathrm{Xe} \rightarrow \mathrm{Ne}_{2}^{+}+\mathrm{Xe}$ & $8.0 \times 10^{-32} \mathrm{~cm}^{6} \mathrm{~s}^{-1}$ & [27] \\
\hline $\mathrm{Ne}^{+}+\mathrm{Ne}+\mathrm{Ne} \rightarrow \mathrm{Ne}_{2}^{+}+\mathrm{Ne}$ & $4.4 \times 10^{-32} \mathrm{~cm}^{6} \mathrm{~s}^{-1}$ & [33] \\
\hline $\mathrm{Ne}^{*}+\mathrm{Xe}+\mathrm{Xe} \rightarrow \mathrm{Xe}_{2}^{*}+\mathrm{Ne}$ & $1.0 \times 10^{-33} \mathrm{~cm}^{6} \mathrm{~s}^{-1}$ & [27] \\
\hline \multicolumn{3}{|l|}{$U V$ radiation } \\
\hline $\mathrm{Xe}_{2}^{*} \rightarrow \mathrm{Xe}+\mathrm{Xe}+\mathrm{h} v$ & $1.0 \times 10^{7} \mathrm{~s}^{-1}$ & [37] \\
\hline $\mathrm{Ne}_{2}^{*} \rightarrow \mathrm{Ne}+\mathrm{Ne}+\mathrm{h} v$ & $8.9 \times 10^{7} \mathrm{~s}^{-1}$ & {$[35]$} \\
\hline $\mathrm{Xe}_{r}^{*} \rightarrow \mathrm{Xe}+\mathrm{h} v$ & $2.9 \times 10^{8} \mathrm{~g} \mathrm{~s}^{-1}$ & {$[36,38]^{\mathrm{d}}$} \\
\hline $\mathrm{Xe}_{r}^{* *} \rightarrow \mathrm{Xe}+\mathrm{h} v$ & $4.05 \times 10^{8} \mathrm{~g} \mathrm{~s}^{-1}$ & {$[36,38]^{\mathrm{d}}$} \\
\hline
\end{tabular}

Note: Units are $\mathrm{cm}^{3} \mathrm{~s}^{-1}$ unless stated otherwise. $T_{\mathrm{e}}$ is the electron temperature $(\mathrm{eV}) . \Delta \varepsilon$ is the change in electron energy as a result of the collision, with negative values indicating a gain in energy.

${ }^{\text {a }}$ Determined as a function of the electron temperature $T_{\mathrm{e}}$ from the lookup table obtained from the solution of the Boltzmann equation.

${ }^{\mathrm{b}}$ Computed through detailed balancing using data from the specified reference.

${ }^{\mathrm{c}}$ Estimated using data from the specified reference.

${ }^{\mathrm{d}}$ Radiation trapped values $-g$ is the radiation trapping factor. 


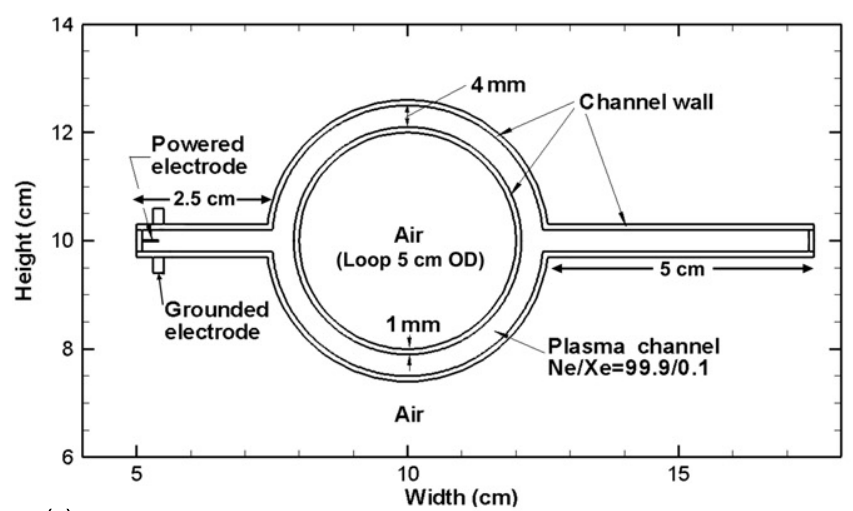

(a)
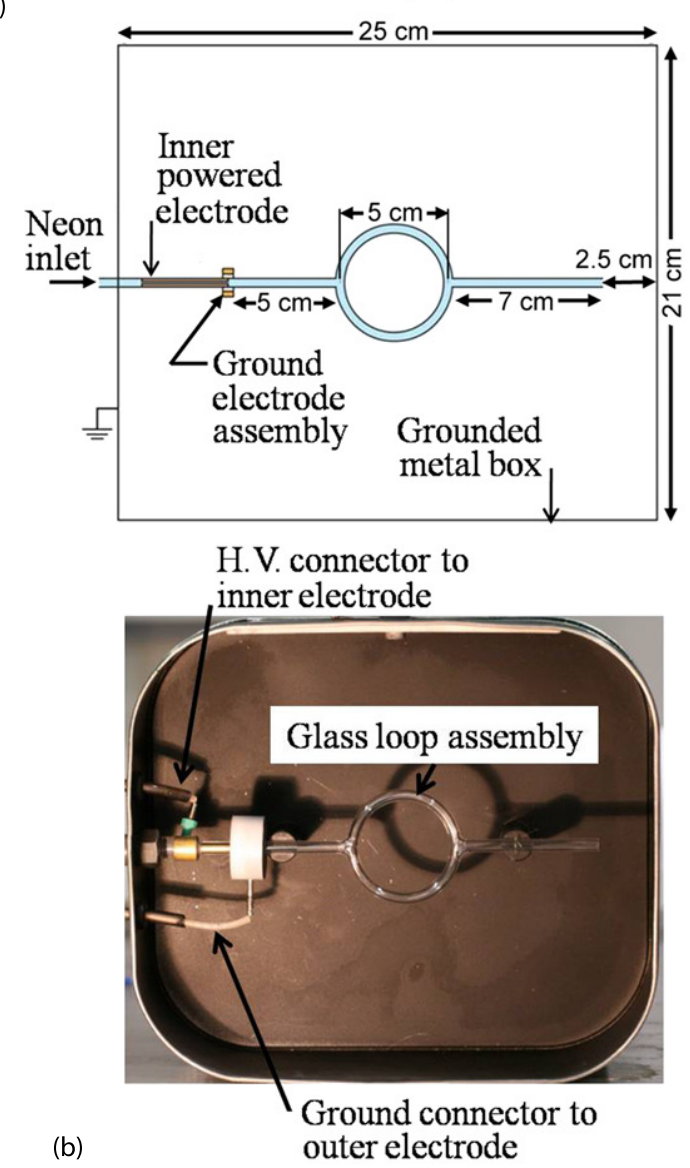

Figure 1. FIW discharge configuration in a branched dielectric channel consisting of a circular loop section and two straight inlet and outlet sections, all surrounded by ambient air. (a) Computational representation. The channel width is $4 \mathrm{~mm}$ and the outside diameter (OD) of the circular section is $5 \mathrm{~cm}$. The full computational domain is a $23 \times 20 \mathrm{~cm}^{2}$ rectangle whose boundaries are grounded. (b) Experimental setup. Top: schematic of the setup including the glass loop assembly, the electrodes (ground assembly and inner powered cathode) and the grounded metal box, with indication of the most relevant dimensions. Bottom: image of the experimental setup, the metal box cover, equipped with the ITO window, was removed.

more closely the model configuration. The plasma jet device, labelled a plasma gun, developed in GREMI has been used to generate neon atmospheric-pressure plasma streams in a loop assembly. The loop assembly consists of the combination of an inlet straight section, a circular section $5 \mathrm{~cm}$ in diameter (measured at the centre of the channel) and an outlet straight section that was used for the final plasma propagation, as shown in figure $1(b)$. The loop is made of borosilicate glass tubes, having a $4 \mathrm{~mm}$ inner diameter and $1 \mathrm{~mm}$ thick walls. A $5 \mathrm{~mm}$ wide ring-shaped outer electrode is set around the inlet section and connected to ground. An additional Delrin ${ }^{\circledR}$ polymer casing encapsulates this outer electrode to prevent undesirable corona discharge and ozone production. The second powered electrode consists of a hollow cylinder, $4 \mathrm{~cm}$ in length with an inner diameter of $1 \mathrm{~mm}$, whose tip is aligned with the centre of the outer ring electrode. A hollow electrode has proven to be the most convenient geometry to produce an axisymmetric discharge reactor, and to achieve an air tight neon feeding connection. The inner electrode is powered with a negative polarity, $25 \mathrm{kV}$ peak amplitude voltage pulse having a rising front of $25 \mathrm{~ns}$. The decay of the voltage pulse occurs over about $10 \mu \mathrm{s}$, so that the voltage amplitude is nearly constant during the first $500 \mathrm{~ns}$ after the peak in voltage, as in the model. A metal box, $25 \mathrm{~cm}$ long in the direction of the inlet to outlet sections, $21 \mathrm{~cm}$ wide in the perpendicular direction and $10 \mathrm{~cm}$ in depth was used to define the ground potential around the loop assembly. Here also, the $25 \mathrm{~cm} \times 21 \mathrm{~cm}$ sizing in the loop plane is close to that considered in the model.

The front surface of the metal box is equipped with an ITO window allowing both for imaging the experiment and for potential grounding. Intensified charge-coupled device (ICCD) images were captured using a PI-MAX3 Roper Scientific camera, equipped with a $50 \mathrm{~mm}$ lens, and driven by a gated pulse allowing for imaging with durations as short as $0.3 \mathrm{~ns}$. In this work, a $1 \mathrm{~ns}$ exposure time was used for imaging the plasma, as a compromise that was made between freezing the position of the FIW and obtaining enough photons to make the exposure. For FIW speeds as high as $10^{8} \mathrm{~cm} \mathrm{~s}^{-1}$, a $1 \mathrm{~ns}$ exposure time results in an uncertainty of position of $1 \mathrm{~mm}$.

Neon (Air Liquide N50) with $99.999 \%$ stated purity from the supplier was used. The impurities include air, $\mathrm{H}_{2} \mathrm{O}$, hydrocarbons, $\mathrm{CO}, \mathrm{CO}_{2}, \mathrm{CF}_{4}$ and $\mathrm{He}$. (See the discussion above concerning choice of gas mixture for the model.) No specific protocol was followed to outgas or evacuate the loop assembly before being flushed with neon at a $0.2 \mathrm{~L} \mathrm{~min}^{-1}$ flow rate. The velocity of the plasma front was measured using an optical fibre bundle. On one side, individual fibres were positioned at different locations along the glass loop and on the other side the bundled fibre outputs were imaged onto the entrance window of a fast rising photomultiplier tube. Each fibre captured a transient optical peak associated with the passing of the plasma ionization front.

Spectroscopic measurements have shown that the plasma emission consists dominantly of the visible atomic neon lines together with slight contributions of molecular bands of nitrogen and hydroxyl radical bands in the UV range, due to small amounts of impurities. Given these results and the detector response, the ICCD imaging dominantly reflects the emission originating from atoms lines of neon.

\section{FIW dynamics in branched tubes and channels}

The complete sequence of the FIW front propagating through the branched dielectric channel is shown, as predicted by the 

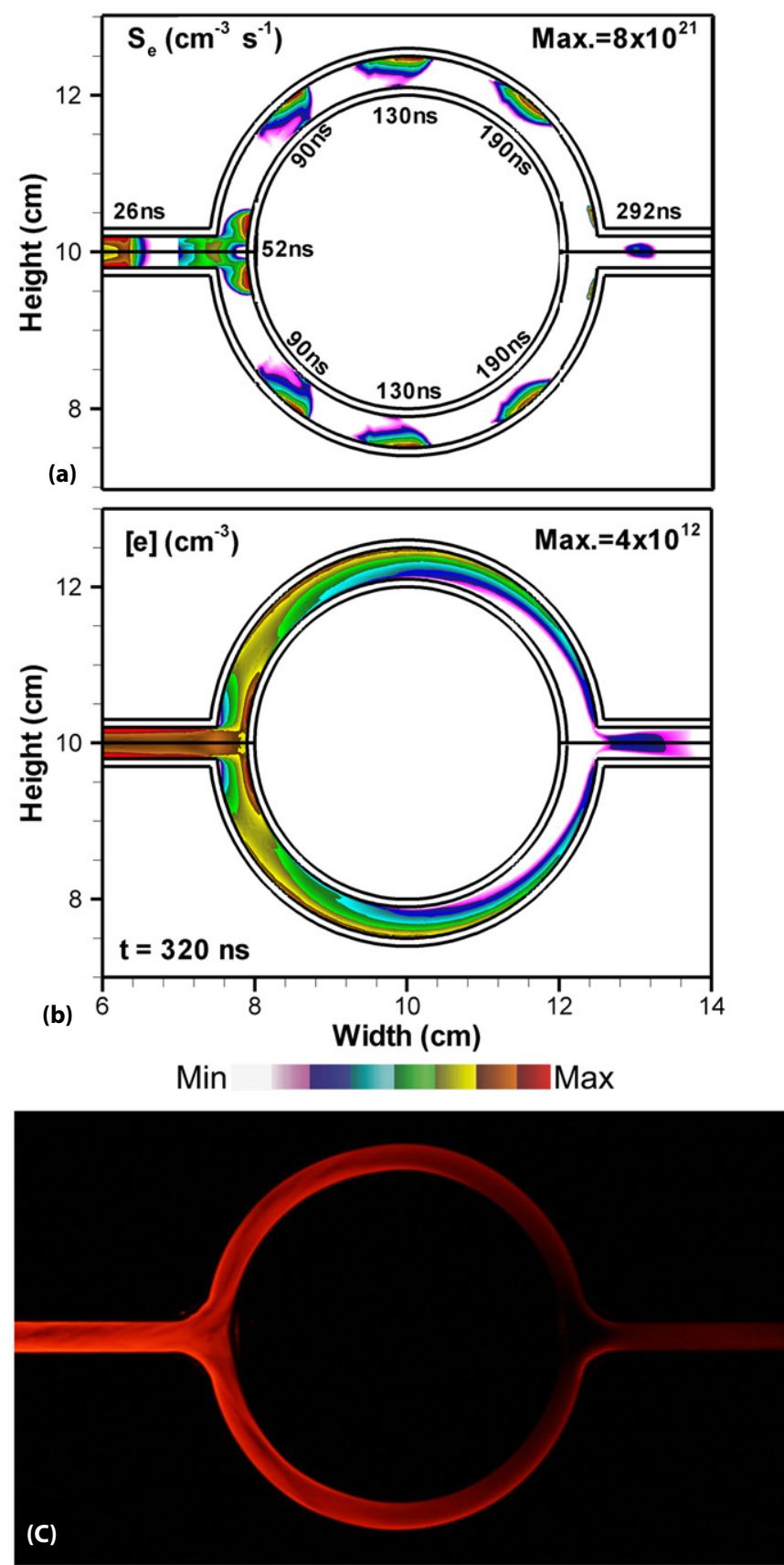

Figure 2. Plasma properties of the FIW front propagating in atmospheric-pressure neon. (a) Computed electron-impact ionization sources at $t=26,52,90,130,190$ and $292 \mathrm{~ns}$.

(b) Computed electron density at $t=320 \mathrm{~ns}$. (c) Experimental time-integrated $16 \mathrm{~ms}$ exposure of visible neon emission. Computed values are over a log scale of three decades with the maximum value noted in each frame.

model, in figure 2. Unless otherwise noted, all computed results are for $\mathrm{Ne} / \mathrm{Xe}=0.999 / 0.001$ mixtures. The ionization front is represented by the rate of electron-impact ionization, $S_{\mathrm{e}}$, that is typically maximum in the large electric fields in the head of the avalanche. $S_{\mathrm{e}}$ is shown at times of 26, 52,90, 130, 190 and $292 \mathrm{~ns}$ after initiation of the discharge. The FIW propagates through the inlet channel ( $26 \mathrm{~ns}$ ) and splits at the inlet-loop junction (52 ns). The speed of the ionization front, $v_{\mathrm{f}}$, is $7 \times 10^{7} \mathrm{~cm} \mathrm{~s}^{-1}$ at the end of the inlet channel. Two FIWs then propagate in the separate legs of the loop channel (90, $130,190 \mathrm{~ns}$ ) while $v_{\mathrm{f}}$ decelerates to $2 \times 10^{7} \mathrm{~cm} \mathrm{~s}^{-1}$. When the two split FIW fronts meet at the loop-outlet junction, they nearly come to a halt, but then they merge and produce a new FIW front in the outlet channel ( $292 \mathrm{~ns})$ which accelerates and reaches a speed of $v_{\mathrm{f}}=3 \times 10^{7} \mathrm{~cm} \mathrm{~s}^{-1}$. These values are within factors of 2 or 3 of the experiments. Sources of the discrepancies are discussed in section 4.

The electron density hugs the wall in the inlet channel, as shown in figure $2(b)$ at $t=320 \mathrm{~ns}$, typical of FIW wave propagation in narrow channels $[11,12]$. This wall-hugging results from the electric field enhancement that occurs at the wall due to the discontinuity in dielectric constant. The electron density at the FIW front is $4 \times 10^{12} \mathrm{~cm}^{-3}$ in the inlet channel. When the FIW splits into the separate legs of the loop, the electron density gradually decreases to $6 \times 10^{11} \mathrm{~cm}^{-3}$ prior to merging, showing a strong mutual influence between the two approaching FIWs. After the launching of the new FIW as a result of the merging of the FIWs from the separate loops, the electron density increases to $9 \times 10^{11} \mathrm{~cm}^{-3}$.

Ionization in the FIW front is dominated by electron impact from the ground state of $\mathrm{Ne}$, which accounts for about $85 \%$ of the total ionization. The remaining contribution comes from Penning ionization and electron-impact ionization from excited states, which account for about $10 \%$ and $5 \%$, respectively. Photoionization at the FIW front is less than $0.1 \%$ that of the electron-impact ionization.

The overall FIW propagation dynamics predicted here can be compared with the experimental observations shown in figures 2 and 3. Snapshots of the plasma emission are shown in figure 3 at different times between 0 and $200 \mathrm{~ns}$ (exposure is $1 \mathrm{~ns}$ ) as the ionization front propagates through the straight and loop sections. The ionization source function shown in figure 2(a) closely resembles the sequence of emission observed in the experiments. The time-integrated neon emission (exposure time $16 \mathrm{~ms}$ ) is shown in figure 2(c). If we assume the density of the optically emitting species is proportional to the electron density, then the electron density distribution shown in figure $2(b)$ is also consistent with the emission image in figure $2(c)$, both showing the decay in the plasma luminescence and the tapering in the plasma volume near the loop-outlet junction. In particular, at the loop-outlet junction, the relatively low emission from the two approaching FIWs and brighter emission from the merged FIW front clearly indicates the enhanced strength of the newly emerged FIW front. This indicates the decreasing rate of ionization, and hence emitting species density, by the mutual influence of the two approaching FIW fronts.

Time-resolved experimental images for emission during the FIW splitting and merging are shown with a higher spatial resolution in figure 4 . The propagation of the FIW in the inlet channel is clearly in the wall hugging mode, as are the two FIWs immediately after the splitting. During the propagation inside the loop, the FIWs start with hugging both walls but gradually shift to hugging only the outer wall. There is a significant reduction in the intensity of the FIWs as they propagate in the loop, evidenced by the much reduced emission intensity when the outlet-loop junction is approached. The 


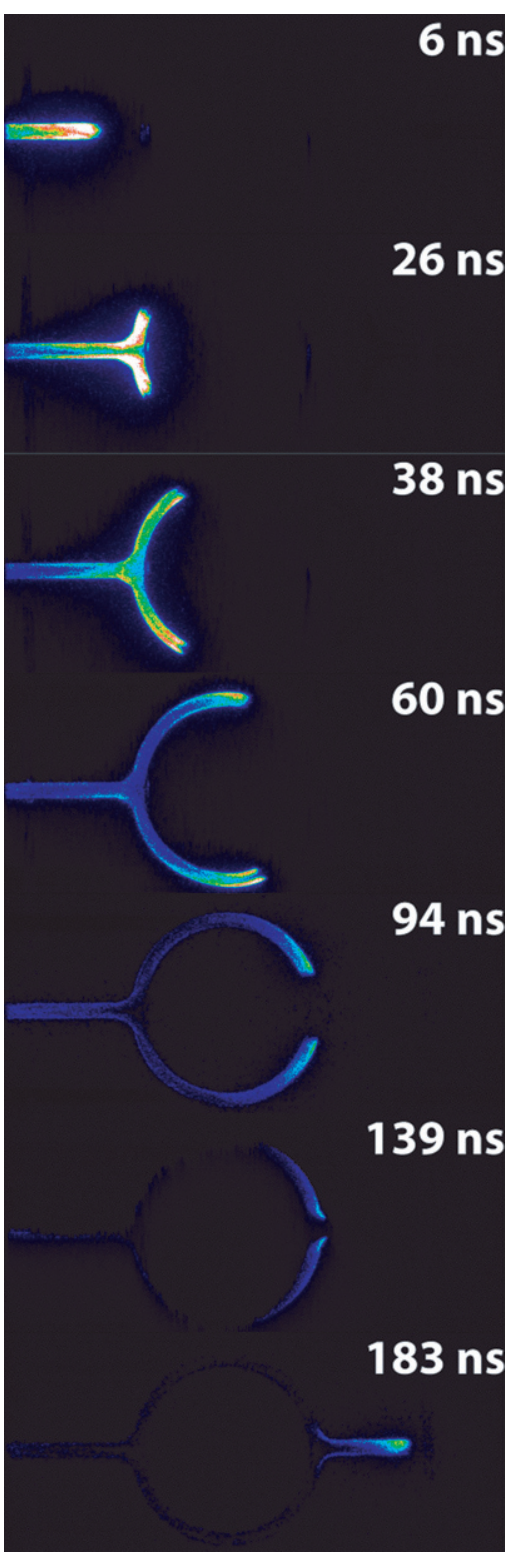

Figure 3. Time-resolved (1 ns exposures) experimental images of emission from atmospheric-pressure neon plasma propagation in a glass capillary including a circular ring.

sequence of the FIW merging at the outlet-loop junction again clearly shows the weakened emission from the two opposing FIW fronts by their mutual influence, while the merged FIW emerging in the outlet channel has a much brighter emission. These trends as predicted by the model are essentially the same.

For the selected experimental conditions shown in figure 3 , the plasma travels in the inlet straight section with a speed of $v_{\mathrm{f}}=4 \times 10^{8} \mathrm{~cm} \mathrm{~s}^{-1}$, arrives at the loop-inlet junction with a speed of $2 \times 10^{8} \mathrm{~cm} \mathrm{~s}^{-1}$ and gradually slows to $3 \times 10^{7} \mathrm{~cm} \mathrm{~s}^{-1}$ at the outlet-loop position. The FIW finally accelerates to $4 \times 10^{7} \mathrm{~cm} \mathrm{~s}^{-1}$ in the outlet straight section after merging of the FIWs. No detailed measurements of the electron density have been performed for the loop experiments. However, preliminary electron density measurements in straight tubes powered with similar voltage waveforms but in helium revealed that the electron density is roughly one order of magnitude higher than that inferred from the simulations for neon xenon
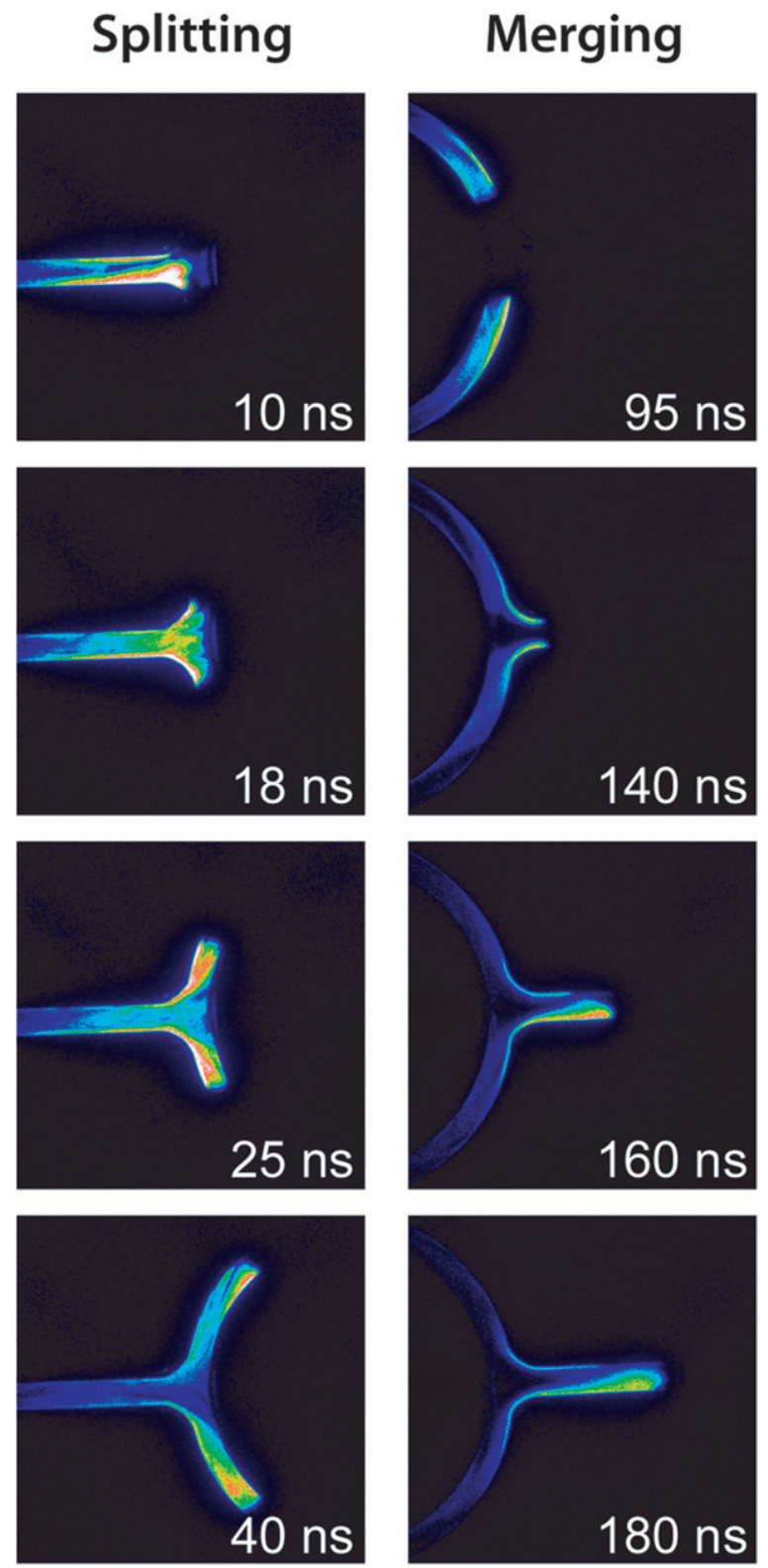

Figure 4. Experimental images of the ionization front (1 ns exposures) showing (left) splitting at the inlet-loop junction and (right) merging at the loop-outlet junction.

mixtures. Experimental measurements, based on hydrogen line broadening, indicate that the electron density is about $7 \times 10^{13} \mathrm{~cm}^{-3}$ in the vicinity of the plasma generation zone and gradually decreases to a few $10^{13} \mathrm{~cm}^{-3}$ a few tens of centimetres downstream.

\subsection{Splitting at the inlet-loop junction}

The computed electron-impact ionization source $S_{\mathrm{e}}$, negative space charge $-\rho$ and electric field $E$ during the propagation of the FIW from the inlet to the splitting loop-junction are shown in figure 5. The corresponding electron temperature $T_{\mathrm{e}}$, electron density $n_{\mathrm{e}}$ and the electric potential are shown in figure 6. Beginning at the powered electrode at about $t=10 \mathrm{~ns}$, the model predicts that the FIW propagates in the inlet channel at a speed about $1 \times 10^{8} \mathrm{~cm} \mathrm{~s}^{-1}$. Approaching 

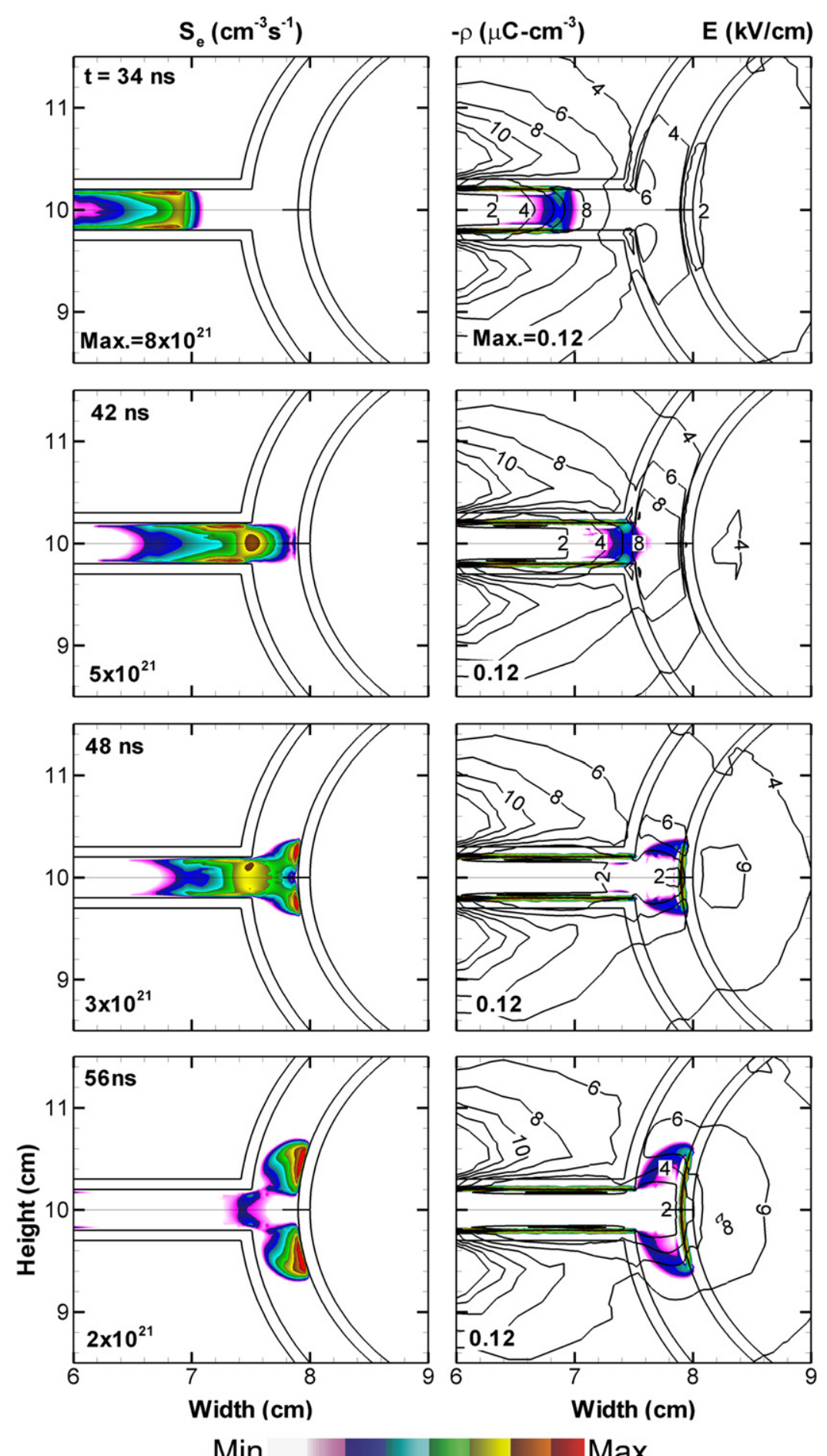

Figure 5. Results from the model for the arrival and splitting of FIW at the inlet-loop junction between (top to bottom) $t=34$ and $56 \mathrm{~ns}$ for $\mathrm{Ne} / \mathrm{Xe}=0.999 / 0.001$. Left: electron impact ionization source function $S_{\mathrm{e}}\left(\mathrm{cm}^{-3} \mathrm{~s}^{-1}\right)$. Right: negative density charge plotted as flood contours and electric field plotted as line contours. The flood contours are on a log scale over three decades with the maximum value noted in each frame.

the inlet-loop junction, the splitting of the incoming FIW front takes place between $t=40$ and $60 \mathrm{~ns}$. First, the propagation of the FIW in the inlet channel is wall-hugging. Both $S_{\mathrm{e}}$ and $n_{\mathrm{e}}$ peak near the wall primarily because of the electric field enhancement at the wall due to the discontinuity in dielectric constant across the surface, and to a lesser extent due to secondary electron emission at the surface with short mean free paths for electron transport [12]. The maximum ionization rate close to the wall is about $8 \times 10^{21} \mathrm{~cm}^{-3} \mathrm{~s}^{-1}$. The negative space charge $-\rho$ is concentrated on the channel wall and in the ionization front where the peak electric field is about $8 \mathrm{kV} \mathrm{cm}^{-1}$ (electric field/gas number density, $E / N=33 \mathrm{Td}$, $1 \mathrm{Td}=10^{-17} \mathrm{~V} \mathrm{~cm}^{2}$ ). The peak electron temperature $T_{\mathrm{e}}$ at $t=34 \mathrm{~ns}$ is in the inlet channel and is about $6.3 \mathrm{eV}$. However, there is a significant heating of electrons diffusing ahead of 

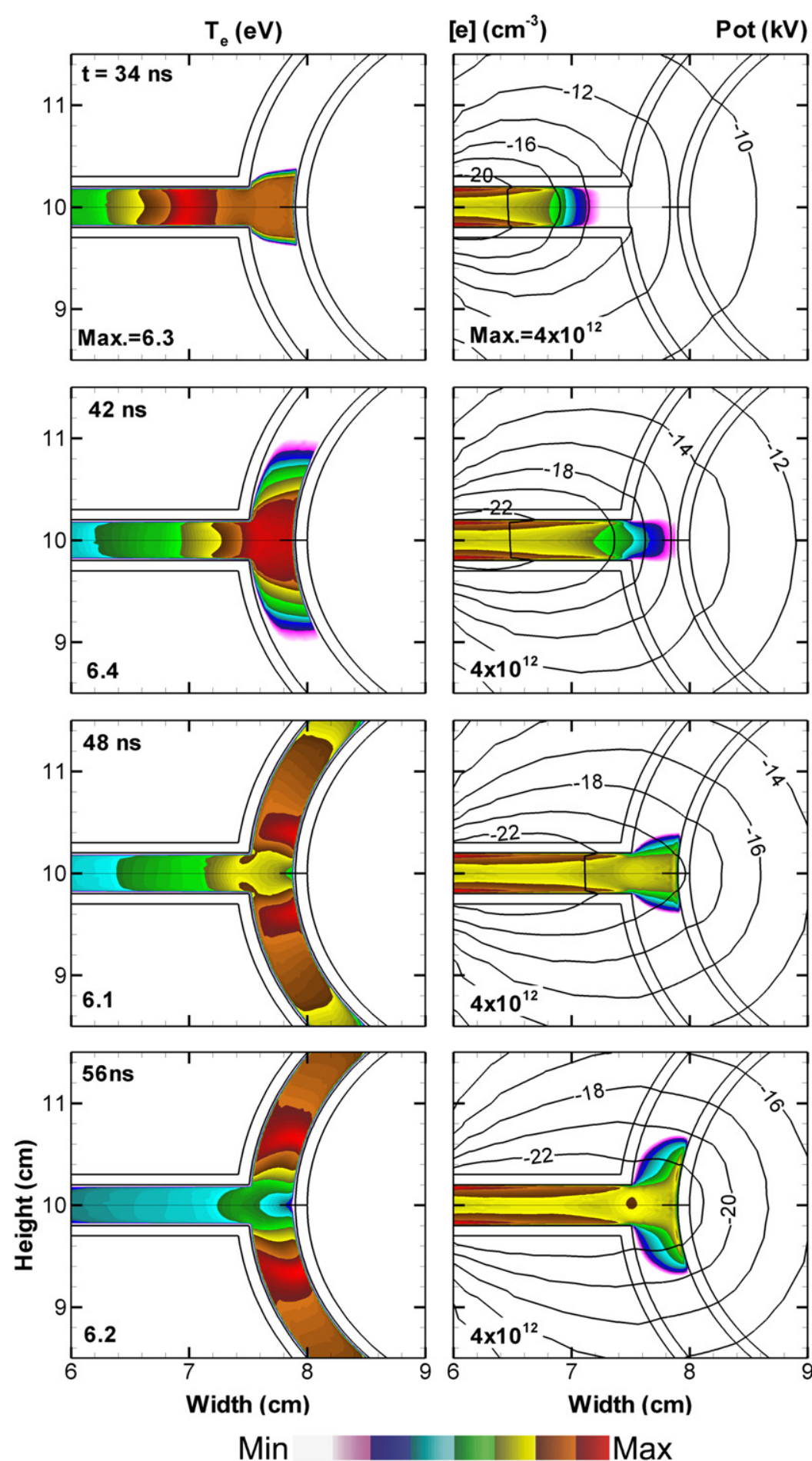

Figure 6. The arrival and splitting of FIW at the inlet-loop junction between (top to bottom) $t=34$ and $56 \mathrm{~ns}$. Left: electron temperature $T_{\mathrm{e}}$. Right: electron density $n_{\mathrm{e}}$ in flood contours and electric potential in line contours. Contours for $T_{\mathrm{e}}$ are on a linear scale and $n_{\mathrm{e}}$ are on a log scale over three decades with the maximum value noted in each frame.

the ionization front in the inlet-loop junction even before the FIW front enters, showing the extension of the intense electric field ahead of the FIW. The electron density is maximum in the wall hugging boundary layer, $n_{\mathrm{e}}=4 \times 10^{12} \mathrm{~cm}^{-3}$ while in the middle of the channel $n_{\mathrm{e}}=6 \times 10^{11} \mathrm{~cm}^{-3}$. This electron density provides sufficient conductivity to largely short out the electric potential, bringing the cathode potential (minus a few $\mathrm{kV}$ dropped across the plasma column) to the ionization front.
As the FIW enters the inlet-loop junction at $t=40 \mathrm{~ns}$, the abrupt change in the channel geometry and the associated change in the spatial distribution of electric field interrupt the FIW propagation along the channel wall. To continue propagation, the wall-hugging FIW front transitions to a localized streamer inside the junction at $t=42 \mathrm{~ns}$. The peak of the ionization shifts from the wall to the centre-line of the channel. This transition is facilitated by electron drift and 
photoionization that seed electrons in the volume in the channel junction and is similar to the exiting of a FIW from a channel or tube into unconstrained gas [22]. The newly formed streamer would, in principle, continue to propagate as a conventional streamer if not for its collision with the inner wall of the loop channel around $t=48 \mathrm{~ns}$. This impingement charges the inner channel wall (see $-\rho$ at $t=42-56 \mathrm{~ns}$ ). This charging is maximum on the centre-line which then produces components of the electric field pointing away from the centre-line (up and down in the figure). The oppositely pointing electric fields produced by the surface charging splits the streamer head into two symmetric, oppositely directed wave fronts. The magnitudes of $S_{\mathrm{e}}\left(3 \times 10^{21} \mathrm{~cm}^{-3} \mathrm{~s}^{-1}\right)$ and $n_{\mathrm{e}}\left(1 \times 10^{12} \mathrm{~cm}^{-3}\right)$ of the two spilt FIWs are smaller compared with the incident FIW. The electric fields in the ionization front are also reduced to about $6 \mathrm{kV} \mathrm{cm}^{-1}(25 \mathrm{Td})$, and so is $T_{\mathrm{e}}$ but by only a few tenths of an $\mathrm{eV}$. The reduction in the electric field, and so reductions in $S_{\mathrm{e}}, T_{\mathrm{e}}$ and $n_{\mathrm{e}}$, are in part a result of the mutual interaction between the two FIWs, to be discussed later.

At $t=56 \mathrm{~ns}$ the two split FIWs begin to propagate in the loop section of the channel. Like their progenitor, these FIWs hug the wall of the channel. $S_{\mathrm{e}}, n_{\mathrm{e}}$ and $-\rho$ mainly hug the inner side of the channel, in large part due to the direction of local electric fields. However, $T_{\mathrm{e}}$ has a relatively large extent across the channel. The conductive, plasma filled inlet channel translates roughly $90 \%$ of the cathode potential to the junction, which produces electric fields which drift electrons into the inner wall. $n_{\mathrm{e}}$ near the outer wall of the loop is low $\left(2 \times 10^{9} \mathrm{~cm}^{-3}\right)$ resulting in the loop channel being only partially filled with plasma. The wall-hugging nature of the plasma in the tubes and the splitting dynamics at the loop-inlet junction are in good agreement with the experimental results shown in figure 4. The most intense emission is observed along the surface of the wall, while there is not a local peak emission clearly seen on the axis.

One discrepancy between the model and experiments is the observation that as the plasma splits from the inlet section into the loop section, the plasma seems to momentarily hug the outer wall before collapsing onto the inner wall. This is not explicitly observed in the results from the model. This discrepancy is attributed to 3D effects that are not captured by the model.

The spitting of the FIW at the T-junction at atmospheric pressure through an intermediate streamer is qualitatively different from such splitting at low pressure. At low pressure (tens of Torr), the FIW front is able to deform and extend across a channel T-junction to make a smooth transition between the inlet plasma wave and the branched plasma waves [39]. We attribute this smoother transition to a higher rate of diffusion of electrons ahead of the ionization front.

\subsection{Propagation in the loop section}

After the splitting of the FIW at the T-junction, the two FIW fronts propagate in the upper and lower halves of the loop channel in essentially a symmetric manner. The structures of the FIW fronts near the inlet-loop junction, near the top of the loop and near the loop-outlet junction, which correspond to $t=70,100,170$ and $232 \mathrm{~ns}$, are shown in figure 7 . Taking advantage of the symmetry, $n_{\mathrm{e}}$ and $T_{\mathrm{e}}$ are shown in the upper half of the figures while $S_{\mathrm{e}}$ and photoionization source function $S_{\mathrm{p}}$ at the same time are shown in the lower half.

At $t=70 \mathrm{~ns}$, the maxima of $S_{\mathrm{e}}$ and $T_{\mathrm{e}}$ in the FIW front have started to shift from the inner to the outer walls of the channel. The peaks of $n_{\mathrm{e}}$ and $S_{\mathrm{p}}$ at this point, however, are still close to the inner wall. By the time the FIW fronts approach the top of the loop channel at $t=100 \mathrm{~ns}$, the transition is essentially complete and all plasma quantities have their maxima near the outer wall. This side-shifting motion is typical for FIW fronts propagating in a curved channel and results from the combined effects of FIW polarity, local electric field orientation and the curvature of the channel [22]. For example, as the FIW front propagates around the circular channel, the direction of the electric field begins to become tangential to the loop, which accelerates the electrons towards the outer channel.

During their propagation around the loop sections, the FIW fronts experience significant deceleration. The FIW speed decreases from $6 \times 10^{7} \mathrm{~cm} \mathrm{~s}^{-1}$ at $t=70 \mathrm{~ns}$ near the inletloop junction to about $1 \times 10^{7} \mathrm{~cm} \mathrm{~s}^{-1}$ at $280 \mathrm{~ns}$ near the loopoutlet junction. The reduction in FIW speed is accompanied by a decreasing ionization rate- the peak value of $S_{\mathrm{e}}$, drops by about 2 orders of magnitude during this time period. In comparison with $S_{\mathrm{e}}$, the magnitude of photoionization $S_{\mathrm{p}}$ is about 2-3 orders of magnitude smaller. Having said that, $S_{\mathrm{p}}$ has a broader nearly channel wide distribution resulting from the quasi-continuous formation of $\mathrm{Ne}_{2}^{*}$ from long-lived metastable states. The end result is that $S_{\mathrm{p}}$ experiences only a modest change in the loop section. $S_{\mathrm{e}}$ is more tightly confined to the head of the FIW. The electron temperature at the FIW front is about $T_{\mathrm{e}}=5-6 \mathrm{eV}$. The peak of $T_{\mathrm{e}}$ is slightly ahead of that of $S_{\mathrm{e}}$, and extends up to a few $\mathrm{cm}$ ahead of the ionization front. For these conditions, the propagation of the negative FIW is driven predominantly by electron drift and diffusion across the FIW front and depends less on photoionization than positive FIW, which is analogous to unconfined streamers.

Reductions in the magnitude of $S_{\mathrm{e}}$ in the head of the FIW and in the speed of the FIW fronts in the loop are determined by at least two factors. The first has to do with the impedance of the plasma. Even for a constant plasma density, as the length of the plasma in the channel increases the impedance increases. The impedance also increases by virtue of the decreasing electron density and decreasing fraction of the cross-sectional area of the channel occupied by the plasma. The plasma region transitions from essentially filling the channel near the inletloop junction to a narrow wall-hugging sheet near the loopoutlet junction. This increased plasma impedance, which is in series with the applied voltage, increases the voltage drop between the cathode and the FIW front, thereby leaving less voltage across the FIW front.

The second and more important factor comes from the mutual influence between the top and bottom FIWs as shown by the electric potential contours in figure 7 . When the two FIW fronts first emerge from the inlet-loop junction, electric potential contours cross through the channel and provide an electric field roughly aligned with the channel axis. This 

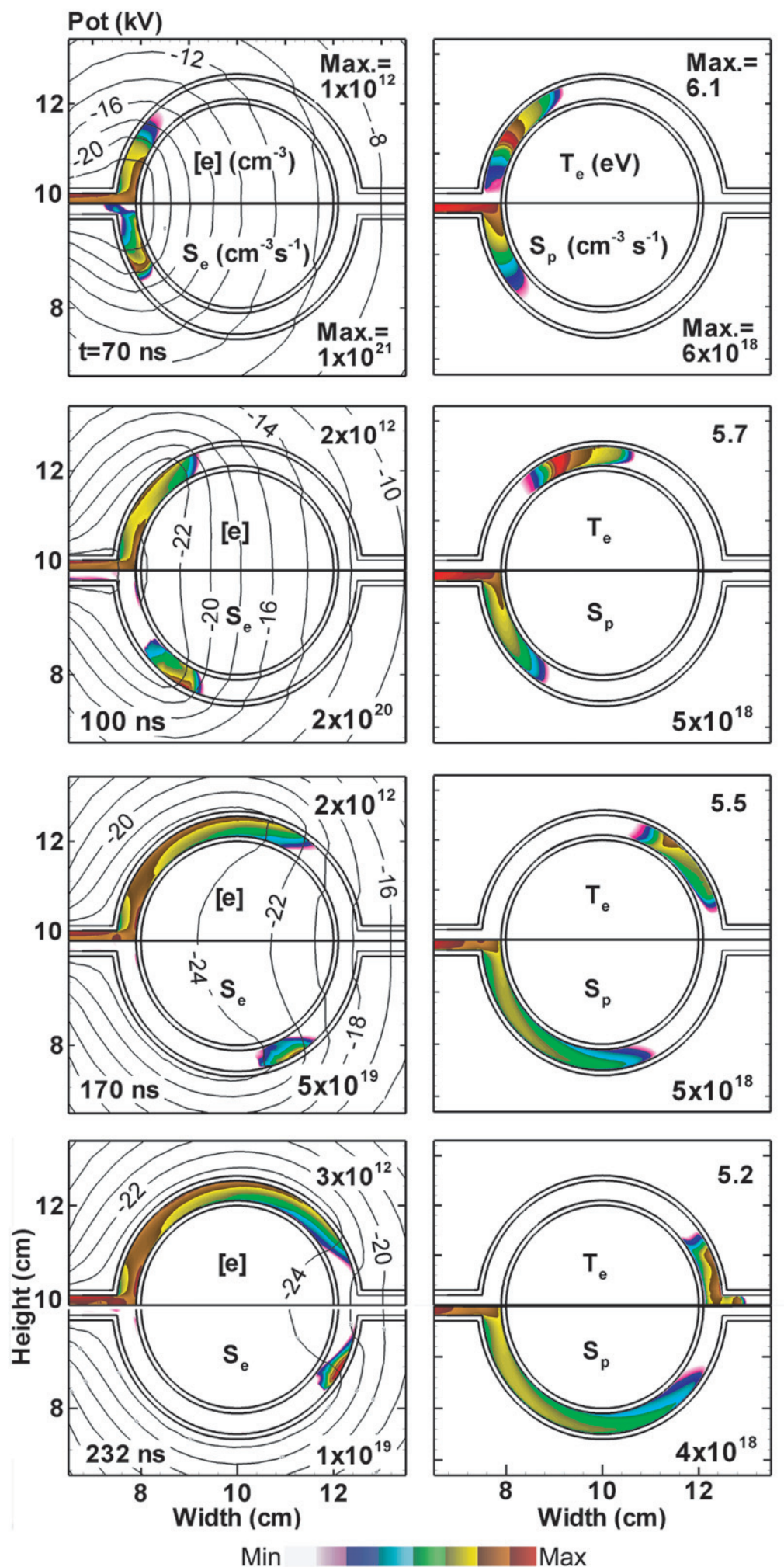

Figure 7. The computed propagation of the (symmetric) FIW fronts inside the loop channel between (top to bottom) $t=70$ and $232 \mathrm{~ns}$. For each time (row), the properties shown in flood images are (upper left) electron density $n_{\mathrm{e}}$, (lower left) electron-impact ionization source $S_{\mathrm{e}}$, (upper right) electron temperature $T_{\mathrm{e}}$ and (lower right) photoionization source, $S_{\mathrm{p}}$. The electric potential is shown in the left column as line contours. Except for $T_{\mathrm{e}}$, the flood contours are a $\log$ scale over three decades with the maximum value noted in each frame.

alignment is facilitated by the walls of the channels ahead of the FIW not being charged—charging which would otherwise shield out the potential. As the FIWs progress around the loop and approach the loop-outlet junction, the FIWs trail behind them a conductive channel and charged walls which shield the electric potential out of the interior of the loop. With this shielding occurring on both top and bottom halves of the loop (mutual interference), there are fewer potential lines which 

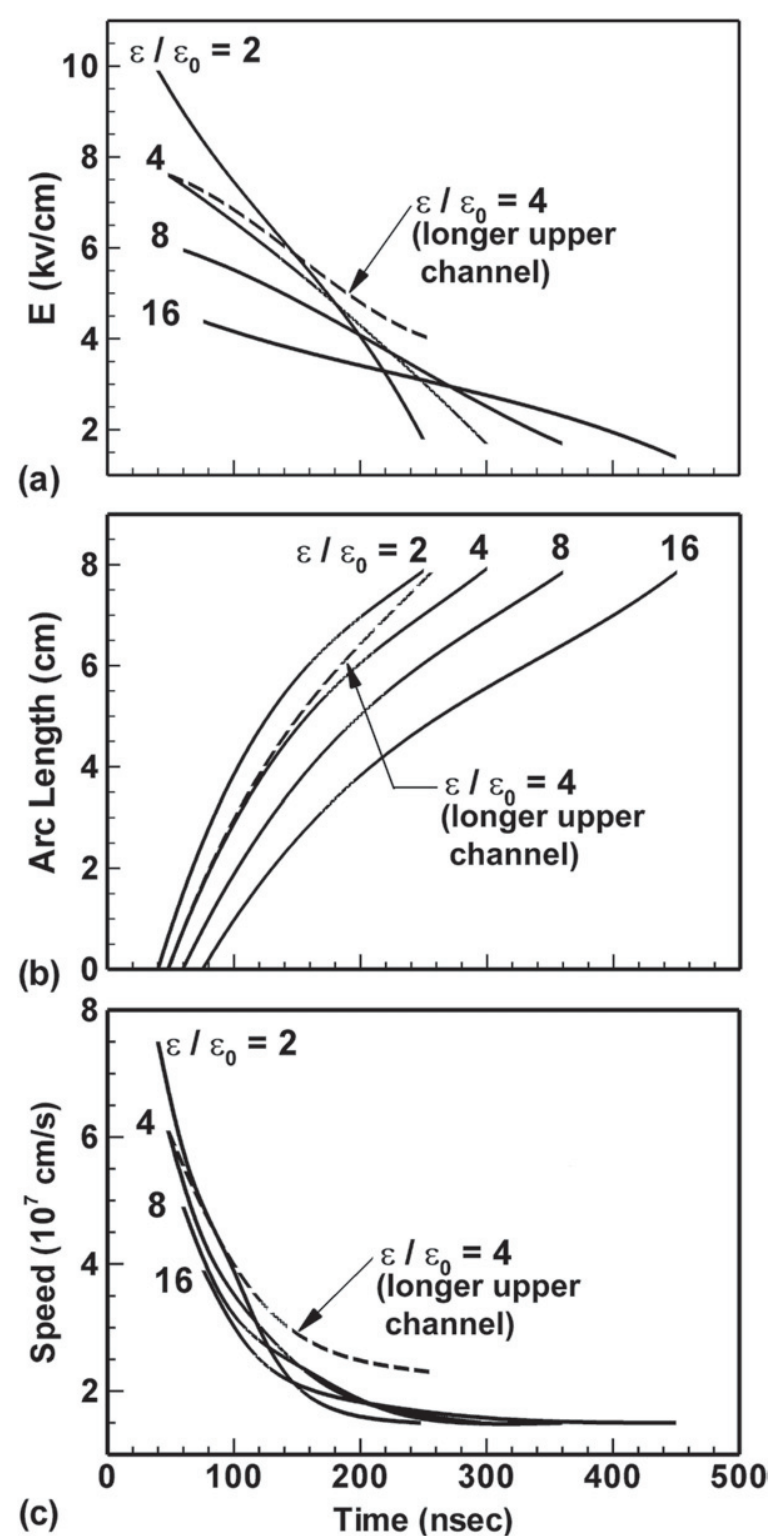

Figure 8. Properties at the leading edge of the FIW for different values of the relative permittivity (dielectric constant). (a) Electric field, $(b)$ arc length traversed by the FIW and $(c)$ speed of the FIW. The dashed lines are for properties in the lower loop in the asymmetric case where the upper loop is longer.

cross the channel and so there is a diminished electric field ahead of the FIW. This effect is exacerbated by the direction of propagation turning nearly perpendicular to the initial direction of the applied electric field. By $t=232 \mathrm{~ns}$, the walls of the channel are charged to within $15^{\circ}-20^{\circ}$ of the junction and the propagation of the two FIWs slows to nearly a stop near the loop-outlet junction because of this mutual influence. At this point, there is virtually no electric component in the direction of propagation.

The maximum electric fields, arc length and ionization wave speed as the FIWs traverse the loop section of the channel are shown in figure 8. Results are shown as a function of time for different dielectric constants, $\varepsilon_{r}$, of the channel wall. The arc length is measured from the inlet-loop junction to the outlet-loop junction over a distance of $8 \mathrm{~cm}$. The delay in starting time for different $\varepsilon_{r}$ is due to the difference in FIW speeds inside the inlet channel. An increasing $\varepsilon_{r}$ leads to a reduced peak electric field and smaller propagation speed because of the increased capacitance and longer charging time of the walls. Although the FIWs for different $\varepsilon_{r}$ start with different speeds and electric fields at the inlet-loop junction, by the time the FIWs approach the loop-outlet junction at $8 \mathrm{~cm}$, their speeds converge to about the same value, $1 \times 10^{7} \mathrm{~cm} \mathrm{~s}^{-1}$, and the electric fields converge to about $2 \mathrm{kV} \mathrm{cm}^{-1}(8 \mathrm{Td})$. This suggests that when the two FIWs approach the merging junction, their properties largely depend upon their mutual influence. That is, the FIWs mutually interact through the shorting of the field which drives their properties to a limiting value.

\subsection{Merging at the loop-outlet junction}

Had there been no outlet channel attached to the loop, the two opposing FIWs of the same polarity would collide and, in a sense, annihilate each other. They would propagate into a region of previously charged channel walls and conductive plasma having a low electric field. At this point, the cathode potential (minus a few $\mathrm{kV}$ voltage drop across the inlet channel) has essentially been translated to the opposite side of the loop from the inlet channel. However, with an outlet channel, the high electric potential brought by the two FIW fronts produces an electric field that is aligned with the axis of the outlet channel. This large electric field $\left(4 \mathrm{kV} \mathrm{cm}^{-1}\right.$ or $\left.16 \mathrm{Td}\right)$, in conjunction with seed electrons produced by electron drift and diffusion, and photoionization, produces a new FIW front in the outlet channel as the two FIWs merge. $S_{\mathrm{e}},-\rho$, and electric field $E$ are shown in figure 9 , and the corresponding $T_{\mathrm{e}}, n_{\mathrm{e}}$, and electric potential in figure 10, as the two FIWs merge and a new FIW is launched in the outlet channel between $t=266$ and $380 \mathrm{~ns}$.

Within $50 \mathrm{~ns}$ of the arrival at the outlet junction of the FIW fronts from the upper and lower legs of the loop, seed electrons start to appear in the outlet channel resulting from both electrons drift and direct photoionization. Due to the shorting of the electric potential in the loop, the majority of the cathode potential is now dropped across the outlet channel, producing an electric field of $3-4 \mathrm{kV} \mathrm{cm}^{-1}(12-16 \mathrm{Td})$ at the beginning of the outlet. This produces significant ionization $\left(S_{\mathrm{e}}=6 \times 10^{18} \mathrm{~cm}^{-3} \mathrm{~s}^{-1}\right)$ with a peak about $5 \mathrm{~mm}$ downstream from the junction, as shown in figure 9 at $t=300 \mathrm{~ns}$. The local, rapidly increasing ionization then produces a new streamer discharge in the outlet channel which propagates with a speed of $3 \times 10^{7} \mathrm{~cm} \mathrm{~s}^{-1}$. This new streamer in the outlet channel is facilitated by the two approaching FIW fronts by increasing the electric field and seeding electrons in the outlet channel, but is not a direct consequence of their merging. In fact, as the streamer in the outlet channel develops between $t=300$ and $334 \mathrm{~ns}$, the two approaching FIW fronts diminish in the loop section rather than turning the corner into the outlet channel. This can be seen more clearly from the contour of the negative space charge $-\rho$ between $t=266$ and $300 \mathrm{~ns}$. The weakening of the approaching FIW at the loop-outlet junction and the launching of the FIW in the outlet channel are also seen experimentally, as shown in figure 4 . 

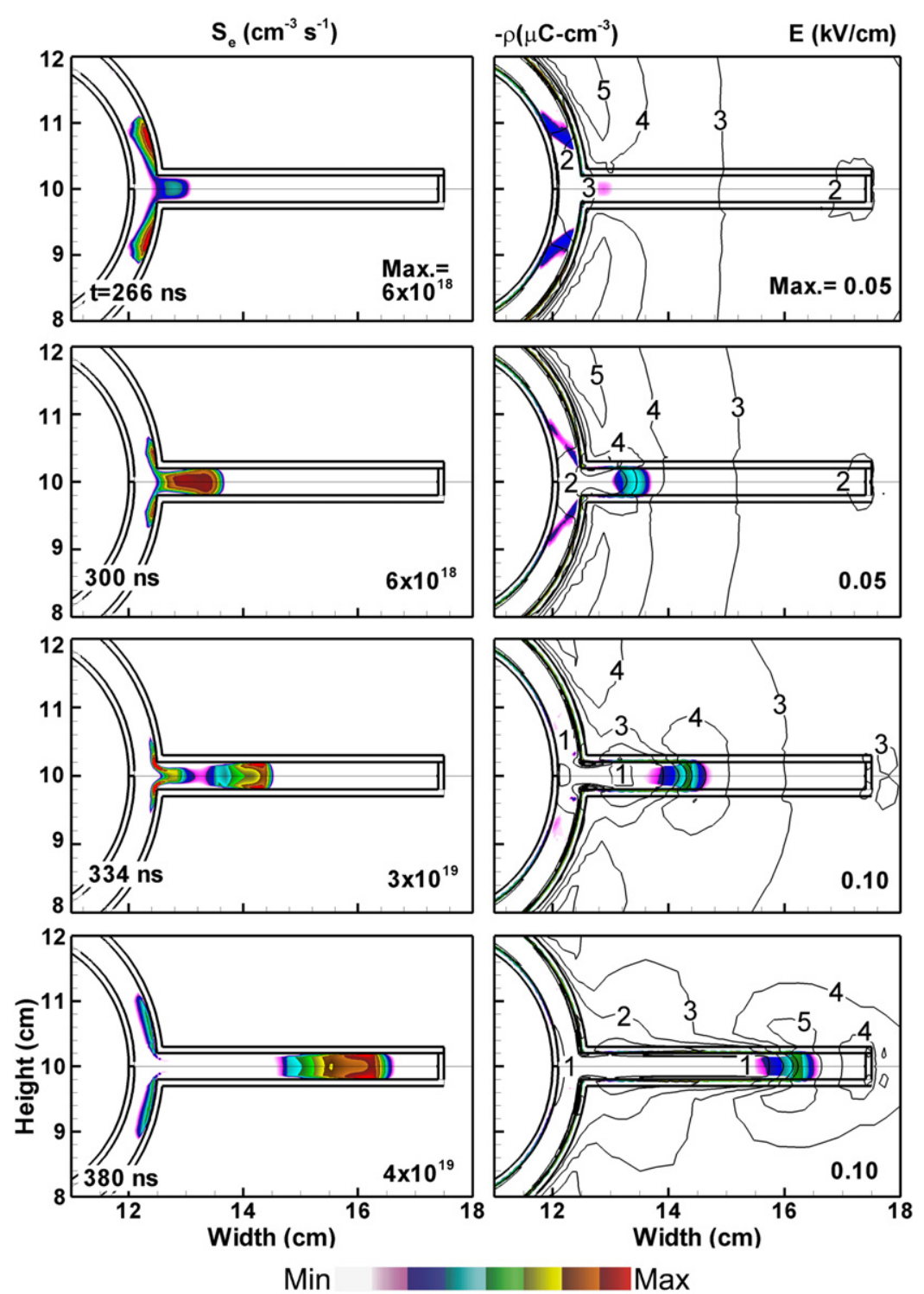

Figure 9. The merging of the two FIW fronts at the loop-outlet junction between (top to bottom) $t=266 \mathrm{~ns}$ and $380 \mathrm{~ns}$. Left electron-impact ionization source function $S_{\mathrm{e}}$. Right: negative space charge and electric field. The flood contours are on a log scale over three decades with the maximum value noted in each frame.

The streamer in the outlet channel begins at a finite distance into the channel, about $5 \mathrm{~mm}$. Once established, the streamer propagates dominantly down the channel away from the loop, but there is also an avalanche that is directed backwards towards the loop-outlet junction, as shown in figure 9 at $t=334 \mathrm{~ns}$. This is, in a sense, another (second-time) splitting of the FIW. This behaviour is similar to a conventional unconstrained streamer discharge initiated in a uniform electric field with a small spot of electrons. Both a positive and negative streamer emerge from the initiating electron cloud. In the outlet channel, the negative space charge at this point concentrates predominantly in the forward travelling front which develops into a wall-hugging FIW front as in the inlet channel. The potential contours show compression of the potential line at this forward moving FIW front. At $t=380 \mathrm{~ns}$, the peak electric field at the forward moving FIW front reaches $5 \mathrm{kV} \mathrm{cm}^{-1}(20 \mathrm{Td})$, about twice the value of the FIWs at their arrival at the loop-outlet junction. The $S_{\mathrm{e}}$ of the merged FIW front is one order of magnitude larger than that of the two FIW fronts entering the junction. This larger electric field and ionization source produce a significant increase in speed, up to $3 \times 10^{7} \mathrm{~cm} \mathrm{~s}^{-1}$, for the merged FIW front in the outlet channel. On the other hand, the backward travelling FIW front experiences an enhanced and diverging electric field as it approaches the loop-outlet junction originating from the two corners of the junction. The backward travelling FIW undergoes another splitting entering into the upper and lower loop channel, primarily in the near-wall plasma regions created by the original two FIWs, as shown in figure 9 around $t=334 \mathrm{~ns}$. Due to the pre-existing electron and excited state density in the upper and lower legs of the loop, the electron density $\left(n_{\mathrm{e}}=9 \times 10^{9} \mathrm{~cm}^{-3}\right)$ produced by the backwards travelling FIW is an order of magnitude higher than their progenitor FIW $\left(n_{\mathrm{e}}=1 \times 10^{9} \mathrm{~cm}^{-3}\right)$. 

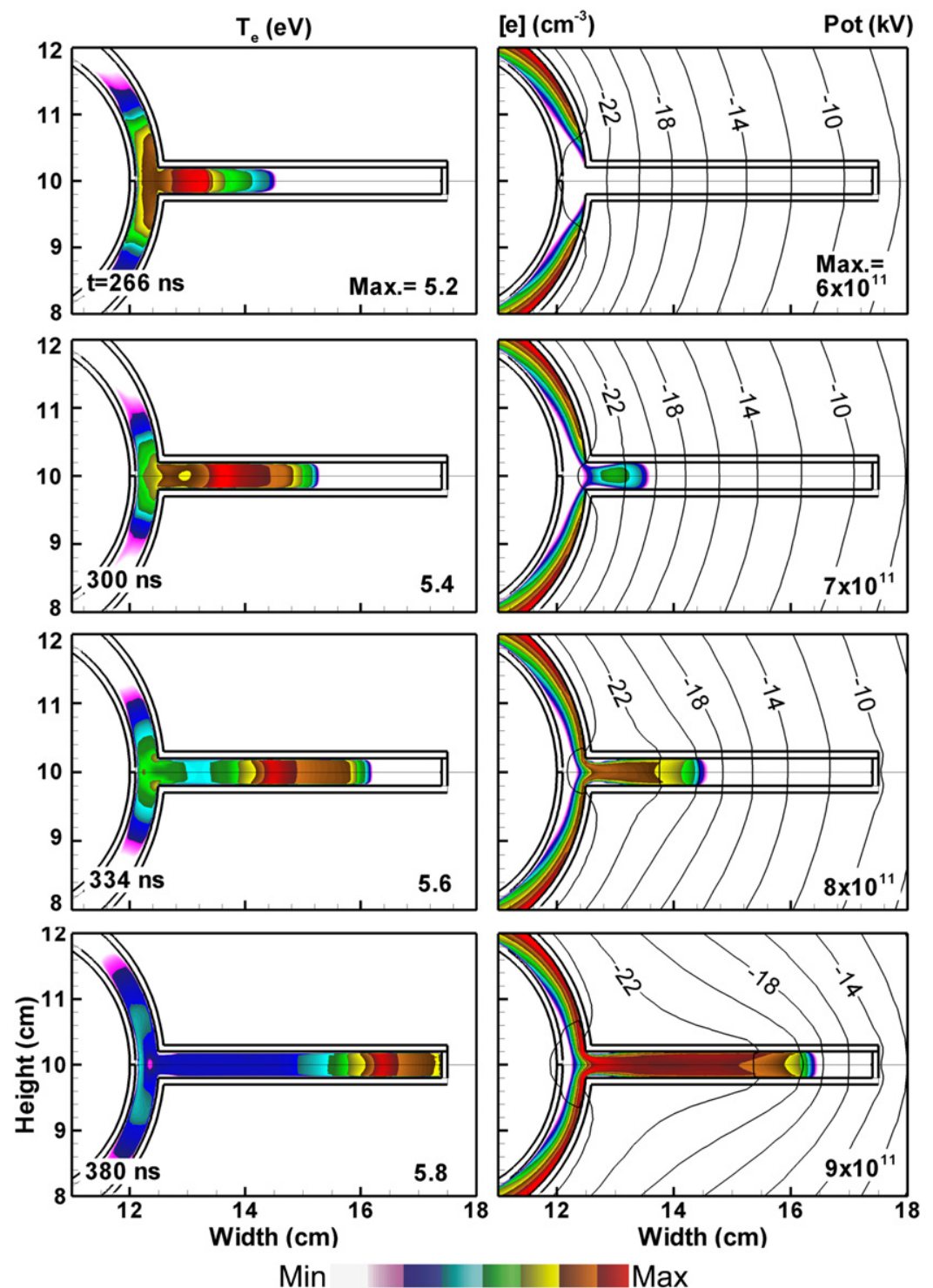

Figure 10. The merging of the two FIW fronts at the loop-outlet junction between (top to bottom) $t=266 \mathrm{~ns}$ and $380 \mathrm{~ns}$. Left: electron temperature $T_{\mathrm{e}}$. Right: electron density $n_{\mathrm{e}}$ and electric potential. $n_{\mathrm{e}}$ is plotted on a log scale over three decades with the maximum value noted in each frame.

These third-time split, backward-travelling ionization waves, though stronger, are short-lived. Once entering the loop channels, they dissipate within a few tens of nanoseconds due to the diminishing potential difference in the existing plasma zones. The appearance of the high ionization region near the junction does not result from the two approaching FIW fronts turning from the loop into the outlet channel, but rather from the merged and then further split streamer heads in the outlet channel travelling back into the loop channel.

The experimental evidence of such back travelling ionization front will require much more detailed analysis of plasma light emission using more sensitive time-resolved detection devices and accumulating data over a larger number of discharge pulses. The electron density enhancement induced by the backward-travelling FIW and the associated electron-impact ionization level are rather low so that they may be hidden by the persistent light emission after merging of the plasma through excitation of metastable levels.

\section{Scaling of the FIW dynamics in branched tubes and channels}

Based on the results of the simulation and the comparison with experimental observations, a model for FIW propagation in symmetric branched channels is proposed as shown in figure 11(a). The time labels are based on the numerical results with the walls having $\varepsilon_{r}=4$ and are used to mark the sequence of the development and to compare the relative FIW speed at different locations. After the first splitting at the inlet-loop junction at around $t=46 \mathrm{~ns}$, it takes about $84 \mathrm{~ns}$ for the first time split FIWs to reach the top of the loop. While to traverse the same distance from the loop top to the outlet junction takes $154 \mathrm{~ns}$, nearly twice as long, indicating a significant deceleration of the FIWs. The merging of the two FIWs takes about $20 \mathrm{~ns}$ and at $t=300 \mathrm{~ns}$ the new streamer emerges in the outlet channel about $5 \mathrm{~mm}$ down the outlet-loop junction. At $t=340 \mathrm{~ns}$, the new streamer has experienced the 

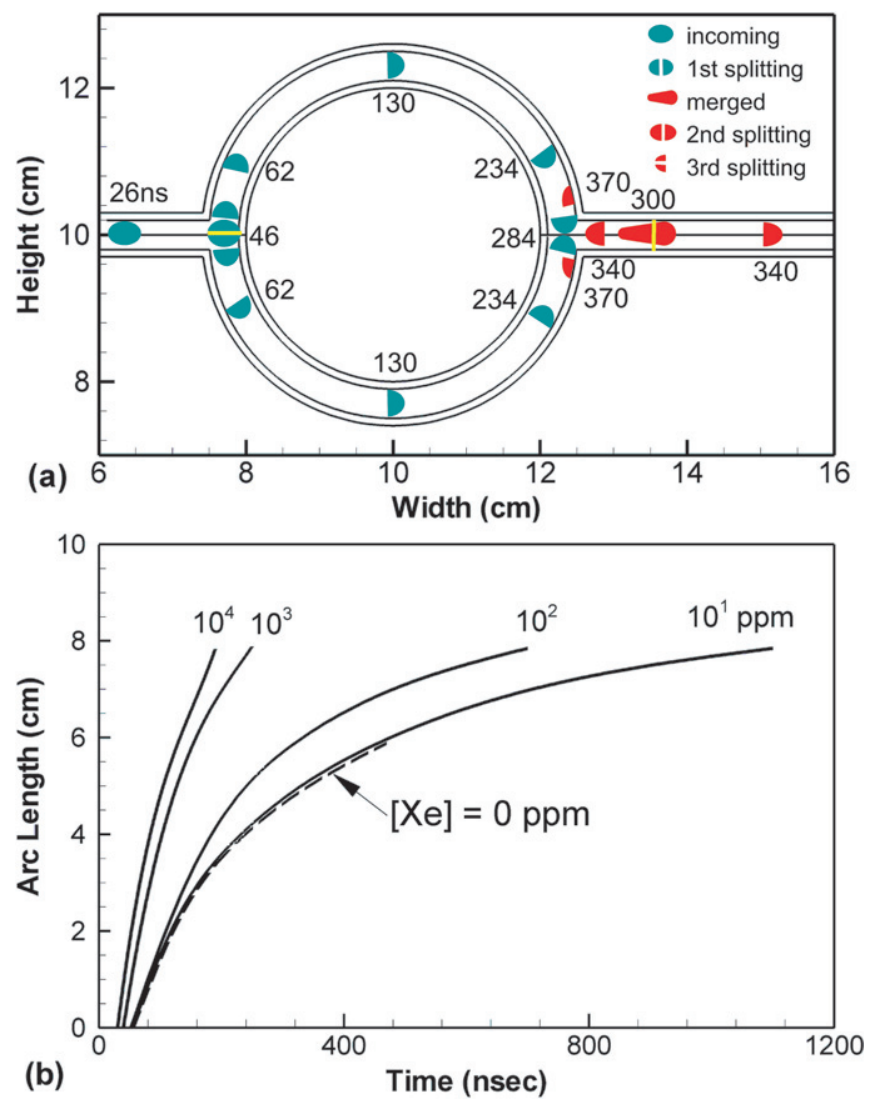

Figure 11. Plasma properties in symmetric loops. (a) A model for splitting and merging FIWs in the symmetric branched circular channel. The time labels are for a FIW in a channel having dielectric constant $\varepsilon_{r}=4$. (b) The distance of traversal of the FIW in the loop section as a function of time for different Xe concentrations for a wall dielectric constant $\varepsilon_{r}=4$. The dashed line is for absolutely pure neon for which the FIW does not reach the loop-outlet junction.

second time splitting, resulting in two streamers propagating in opposite directions. The back travelling second-time split streamer reaches the junction and at $t=370 \mathrm{ns,} \mathrm{it} \mathrm{splits}$ the third time and forms two FIWs propagating back into the loop channel. While these third-time split FIWs quickly disappear in the loop channel, the second-time split, forward travelling streamer develops into a new wall-hugging FIW in the outlet channel. The cases for other dielectric constants are qualitatively similar. We note that this FIW propagation model in a branched channel is based on results from $2 \mathrm{D}$ simulations. The FIW dynamics in truly $3 \mathrm{D}$ channel and tube junctions are more complex and remains a subject of future studies.

In the experiments, the glass tubes of the loops are circular cylinders as opposed to the 2D planar channels used in the model. To help quantify the differences between the FIWs propagating in tubes and channels, simulations were performed using only a straight tube with the same the inlet and outlet as described above. The straight section is either a channel (2D Cartesian) or a cylindrically symmetric tube. The length of the straight section is $12 \mathrm{~cm}$, the diameter (or width) is $4 \mathrm{~mm}$ and the wall thickness is $1 \mathrm{~mm}$. Reflective boundary conditions (that is, zero spatial gradient) were used on the centre-line for the 2D, Cartesian channel.
Comparisons of the FIW properties in the cylindrical tube and planar channel are shown in figure 12(a) and $(b)$ at $t=80 \mathrm{~ns}$. The electron density $n_{\mathrm{e}}$ is plotted in the upper half of the figure and the corresponding ionization source function $S_{\mathrm{e}}$ is plotted in the lower half. At this time, the FIW front is $6 \mathrm{~cm}$ downstream of the inlet for the tube, and $4 \mathrm{~cm}$ for the channel. The wall-hugging nature of the FIW propagation is qualitatively the same in both the tube and channel, and in fact is more pronounced in the tube. The maximum values of both $n_{\mathrm{e}}\left(2 \times 10^{13} \mathrm{~cm}^{-3}\right)$ and $S_{\mathrm{e}}\left(4 \times 10^{21} \mathrm{~cm}^{-3} \mathrm{~s}^{-1}\right)$ are about one order of magnitude higher in the tube than that in the channel, indicating an enhanced plasma focusing effect provided by the stronger confinement in the tube.

The maximum value of the electric field $E$ at the FIW front and the speed $v_{\mathrm{f}}$ as a function of FIW traversal distance are also shown in figure 12. Throughout the propagation, both $E$ and $v_{\mathrm{f}}$ in the tube are higher than that in the channel by about a factor of 2. The FIW in the tube also shows an acceleration phase before $d=6 \mathrm{~cm}$ while in the channel the FIW basically decelerates monotonically. The end result is that for otherwise identical conditions, $v_{\mathrm{f}}$ is about a factor of 2 larger in the tube than the channel. Experimental measurements of $v_{\mathrm{f}}$ are approximately a factor of 2 larger than predicted by the model. These differences are likely a consequence of approximating the truly cylindrical tubes and 3D junctions with 2D channels.

These geometric factors are also likely responsible for the small differences in the patterns of the plasma during the splitting at the inlet-loop section between the experiment and model, as well as a few second order effects. For example, in the model, the amplitude of the cathode voltage is kept constant after the ramp-up period while in the experiment there is a small gradual decrease in the voltage across the electrodes that is associated with the impedance variation of plasma. The predicted plasma propagation dynamics are nevertheless in good agreement with the measurements. For example, in the experiment, it takes twice as long for the FIW to propagate from the top of the loop to the outlet compared with the time required to reach the top of the loop from the inlet. This is essentially the same as predicted by the model.

In the experiments, pure $\mathrm{Ne}$ gas (stated impurity level $<30 \mathrm{ppm}$ ) was used to flow through the tube. In the model if absolutely pure $\mathrm{Ne}$ is used, the split FIW fronts in the loop channels did not reach the outlet-loop junction and no merged ionization front is produced. However, for the same voltage pulse, inclusion of a trace amount of a lower ionization potential gas, such as Xe, leads to an increased ionization and a merging of the FIW. A series of simulations were carried out while varying the Xe impurity level from $0 \%$ to $1 \%$. The trajectories of the split FIWs inside the loop channel are shown in figure 11(b). An increasing concentration of the Xe impurity produces a higher FIW speed, due to both an increase in electron impact ionization and photoionization. For absolutely pure $\mathrm{Ne}$, the FIWs only reach about three quarters of the loop length. Despite the quantitative difference in speeds, the qualitative features of splitting and merging dynamics remain the same as those described above.

Many of the previously discussed dynamics of the dualFIWs result from their symmetry. A natural extension of 

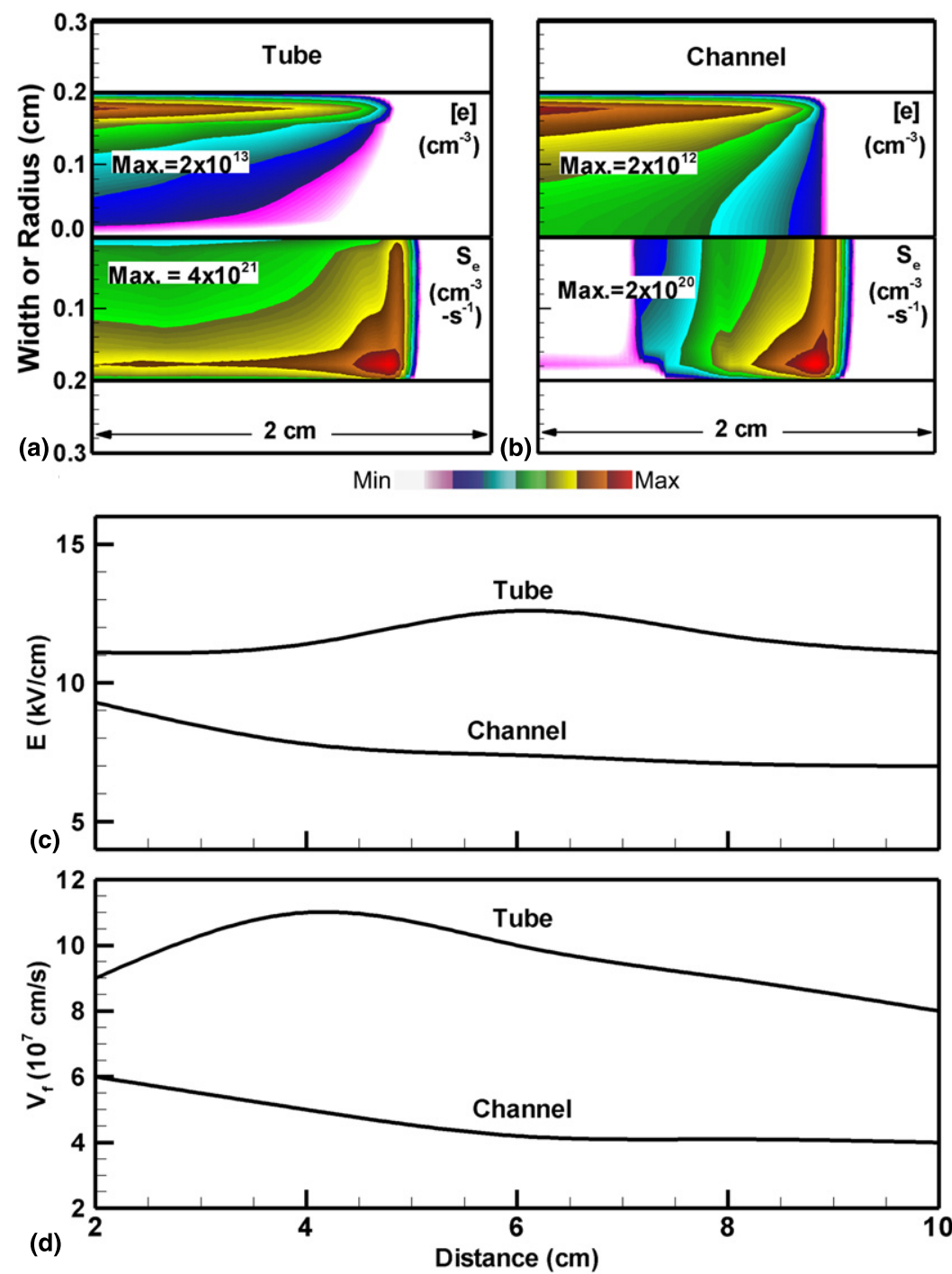

Figure 12. Comparisons between FIW discharges in a cylindrical tube and a planar channel. The electron density $n_{\mathrm{e}}$ (upper half) and electron-impact ionization $S_{\mathrm{e}}$ (lower half) at the FIW front at $t=80 \mathrm{~ns}$ are shown in a $(a)$ tube and $(b)$ channel. Properties at the head of the FIW are shown for propagation distance along the tube or channel for $(c)$ electric field and $(d)$ propagation speed. $n_{\mathrm{e}}$ and $S_{\mathrm{e}}$ are plotted on a $\log$ scale over three decades with the maximum value noted in each frame.

the present discharge configuration is to break the mirror symmetry. This can be done in several ways, for example, using different channel lengths, using materials with different dielectric constants between the upper and lower loop sections, or changing the junction locations so that inlet and outlet channels are not aligned.

An example of propagation of FIW in an asymmetric channel loop is shown in figure 13( $a$ ) where $S_{\mathrm{e}}, n_{\mathrm{e}}$ and potential are plotted as a function of time. The discharge conditions are the same as for the examples discussed above except that the upper loop channel is 1.5 times longer than the lower half. After the FIW splitting at the inlet-loop junction, the lower FIW front arrives at the loop-outlet junction earlier than the FIW in the upper loop. In fact as shown in figure 8, the lower FIW has a higher peak electric field and travels even faster than in the symmetric case due to the reduced influence from the upper FIW. The early arrival at the loop-outlet junction of the lower FIW front basically forestalls the propagation of upper FIW, preventing it from reaching the junction. The lower FIW charges its channel and so shields out the electric potential, which reduces the potential difference across the FIW in the upper loop. The FIW in the upper loop slows and eventually stalls. However, even in the absence of the slower FIW, the earlier arriving FIW front is still capable of inducing a new streamer in the outlet channel, as shown at $t=340 \mathrm{~ns}$, which eventually develops into a FIW front travelling in the outlet channel.

The interaction of asymmetric FIW was also studied experimentally, as shown in figure $13(b)$. This image was obtained by producing an asymmetry in the ground potential by placing a grounded metal rod near the lower branch of the circular section. This increases the speed of the propagation of the FIW in the lower loop compared with the upper loop. As predicted by the model, plasma generation in the outlet straight section together with the 'second splitting' of the FIW from the lower branch are observed in the experiment. The experimental also shows backwards propagation of the split ionization wave from the lower channel into the upper branch, 

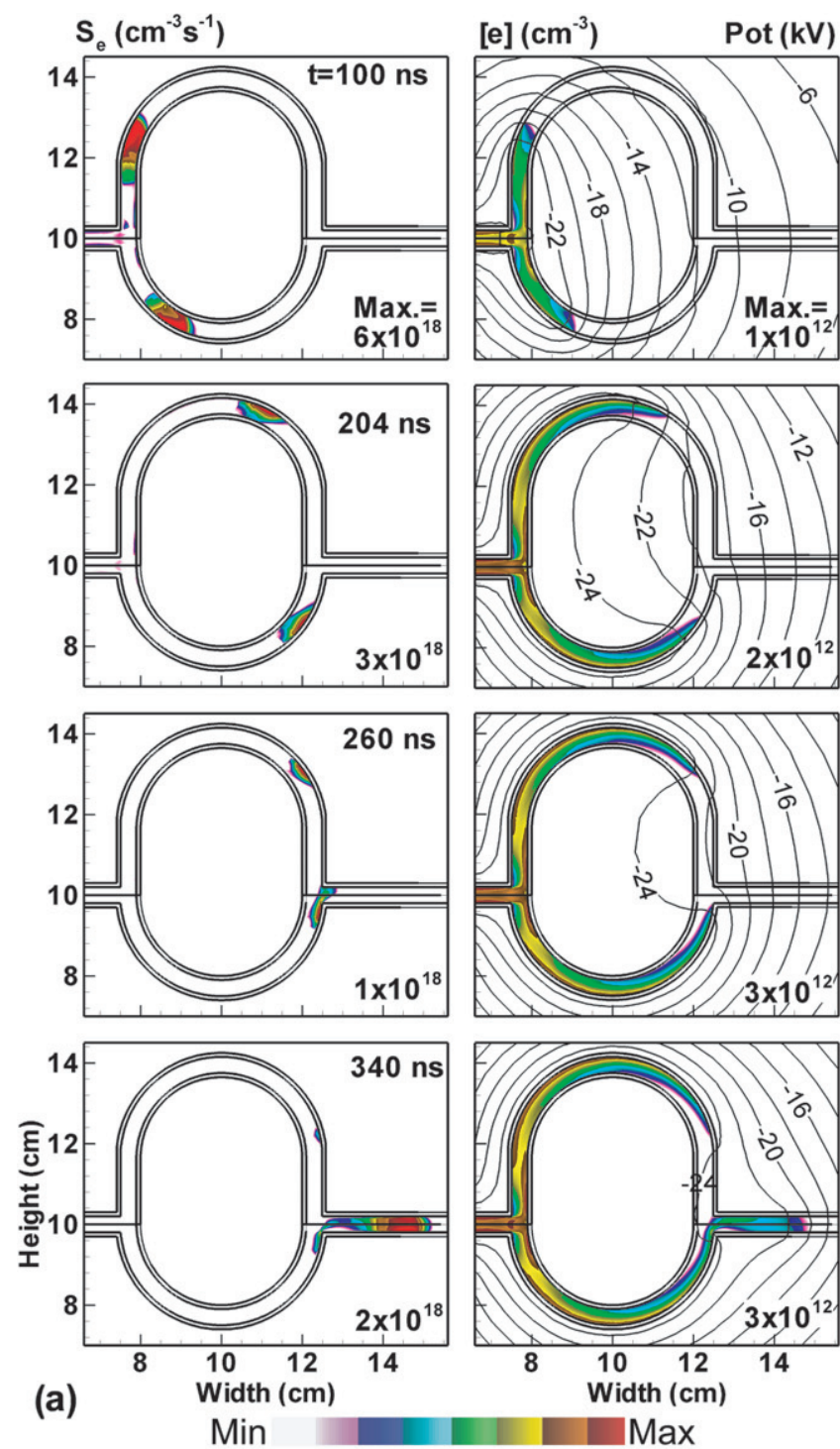

(a)

$$
\text { Min }
$$

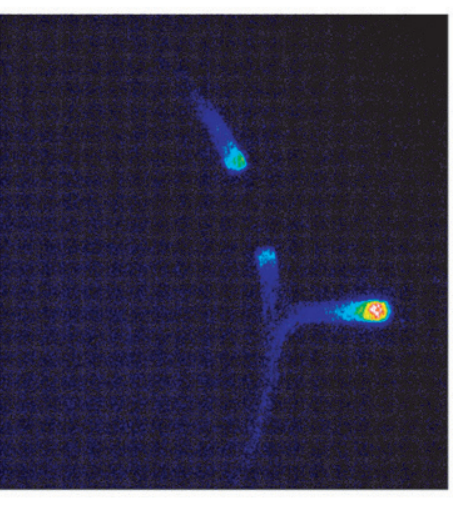

(b)

Figure 13. FIW properties in an asymmetric loop. (a) Computed values for times (top to bottom) of $t=100 \mathrm{~ns}$ to $340 \mathrm{~ns}$. Left: electron-impact ionization source $S_{\mathrm{e}}$. Right: electron density $n_{\mathrm{e}}$ and electric potential. The upper half of the loop channel is 1.5 times longer than the lower half. The lower FIW arrives at the junction first, which stalls the FIW in the upper loop, and also induces a new FIW in the outlet channel. The contours are on a log scale over three decades. (b) Experimental imaging ( $1 \mathrm{~ns}$ exposure) of the plasma merging at the outlet-loop section for conditions where the plasma propagating in the lower branch of the loop arrives at the junction first. $n_{\mathrm{e}}$ and $S_{\mathrm{e}}$ are plotted on a log scale over three decades with the maximum value noted in each frame. at which time both FIWs stall. This latter plasma experiences strong mutual influence from the plasma travelling in the upper branch. The end result is that both FIWs dissipate as described in the modelling for the case of a loop without an outlet section.

No detailed studies of the role of the pulse repetition rate on plasma dynamics during propagation of the FIW in the loop assembly have been performed. However, recent work has addressed the role of pulse repetition rate on ionization front velocity in atmospheric-pressure neon in straight tubes and the expansion of the plasma into ambient air at the end of the tube $[11,40]$. For a given combination of the peak voltage amplitude and gas flow rate, the length of the plasma expansion beyond the tube decreases by a factor of two when the pulse repetition rate increases from single shot operation to $75 \mathrm{~Hz}$. Further increases in the repetition rate to $350 \mathrm{~Hz}$ did not affect the plasma expansion. The decrease in the length of the plasma plume going from 1 to $75 \mathrm{~Hz}$ likely resulted from charging of the inner capillary wall and subsequent shielding of the electric potential. For repetition rates below $75 \mathrm{~Hz}$, the charge deposition on the inner capillary surface is continuously enhanced as the pulse repetition rate increases, thus gradually reducing the plasma propagation length. For repetition rates higher than $75 \mathrm{~Hz}$, it is likely that a steady state is achieved between charge deposition and recombination processes. Surprisingly, the ionization front velocity was independent of the pulse frequency over this same range [40]. It appears that the pulse to pulse residual volume preionization is sufficiently small at these low repetition rates (and long interpulse periods) as to not significantly affect the FIW speed.

\section{Concluding remarks}

Two-dimensional numerical simulations were performed of FIW discharges initiated by a nanosecond, high-voltage pulse and propagating in a branched channel consisting of two straight inlet and outlet sections separated by a circular loop section. Comparisons were made with experiments in which images of the FIW in the channels were obtained with ns resolution. The channel is filled with $\mathrm{Ne}$ at atmospheric pressure with trace amount of impurity represented by Xe. The predicted dynamics of the FIW splitting, propagation and merging are found to agree qualitatively with the experimental observations. The propagation of FIW in the channel tends to be in a wall-hugging mode. For straight channels, the FIW develops along both walls while for curved channels the plasma typically hugs one side of the wall, but can shift from one side to the other during the propagation. The splitting of the FIW approaching a T-intersection is facilitated by charging of the opposing wall, which produces lateral electric fields that initiate streamers in the opposite directions. The merging of the FIW at the outlet junction is facilitated through a newly formed, intermediate streamer, which results from high electric field brought by the approaching FIWs and enhanced locally by the junction geometry.

There is a significant reduction in FIW ionization sources and speed during the propagation in the channel, particularly near the outlet-loop junction due to the mutual influence between the FIWs. The shorting and shielding of the electric 
potential due to the charging of the walls and conductivity of the plasma reduces the electric field into which the other FIW propagates. For symmetric systems, this results in a lower speed for both FIWs. In asymmetric systems where one FIW progresses faster (or further) along the loop towards the outlet junction, the leading FIW can short (or shield) the potential to such a degree that the trailing FIW will stall. The merging and subsequent propagation of FIW into the outlet channel is not necessarily a continuous process. The merged FIW may seed a new streamer in the outlet channel which then go through further splitting process to produce forward and backwardtravelling FIWs.

\section{Acknowledgments}

This work at the University of Michigan was supported by the United States Department of Energy Office of Fusion Energy Science. The GREMI laboratory is supported through APR Région Centre 'Plasmed' and ANR Blanc 'PAMPA'. V Sarron is supported by Conseil Général du Loiret.

\section{References}

[1] Vasilyak L M, Kostyuchenko S V, Kudryavtsev N N and Filyugin I V 1994 Fast ionisation waves under electrical breakdown conditions Phys.-Usp. 37 247-69

[2] Lagarkov A N and Rutkevich I M 1994 Ionization Waves in Electrical Breakdown of Gases (New York: Spinger) chapter 3-5

[3] Starikovskaia S M, Anikin N B, Pancheshnyi S V, Zatsepin D V and Starikovskii A Y 2001 Pulsed breakdown at high overvoltage: development, propagation and energy branching Plasma Sources Sci. Technol. 10 344-55

[4] Adamovich I V, Choi I, Jiang N, Kim J-H, Keshav S, Lempert W R, Mintusov E, Nishihara M, Samimy M and Uddi M 2009 Plasma assisted ignition and high-speed flow control: non-thermal and thermal effects Plasma Sources Sci. Technol. 18034018

[5] Baksht E Kh, Burachenko A G and Tarasenko V F 2009 UV lasing in nitrogen pumped by a runaway-electron-preionised diffuse discharge Quantum Electron. 39 1107-11

[6] Stoffels E, Sakiyama Y and Graves D 2008 Cold atmospheric plasma: charged species and their interactions with cells and tissues Trans. Plasma Sci. 36 1441-57

[7] Walsh J L, Iza F, Janson N B, Law V J and Kong M G 2010 Three distinct modes in a cold atmospheric pressure plasma jet J. Phys. D: Appl. Phys. $\mathbf{4 3} 075201$

[8] Mericam-Bourdet N, Laroussi M, Begum A and Karakas E 2009 Experimental investigations of plasma bullets J. Phys. D: Appl. Phys. 42055207

[9] Teschke M, Kedzierski J, Finantu-Dinu E, Korzec D and Engemann J 2005 High-speed photographs of a dielectric barrier atmospheric pressure plasma jet Trans. Plasma Sci. 33 310-1

[10] Kim J Y, Kim S, Wei Y and Li J 2010 A flexible cold microplasma jet using biocompatible dielectric tubes for cancer therapy Appl. Phys. Lett. 96203701

[11] Robert E, Barbosa E, Dozias S, Vandamme M, Cachoncinlle C, Viladrosa R and Pouvesle J M 2009 Experimental study of a compact nanosecond plasma gun Plasma Process. Polym. 6 795-802

[12] Jánský J, Le Delliou P, Tholin F, Tardiveau P, Bourdon A and Pasquiers S 2011 Experimental and numerical study of the propagation of a discharge in a capillary tube in air at atmospheric pressure J. Phys. D: Appl. Phys. 44335201

[13] Pothiraja R, Bibinov N and Awakowicz P 2011 Amorphous carbon film deposition on the inner surface of tubes using atmospheric pressure pulsed filamentary plasma source J. Phys. D: Appl. Phys. 44355206

[14] Mrad O, Saunier J, Aymes Chodur C, Rosilio V, Agnely F, Aubert P, Vigneron J, Etcheberry A and Yagoubi N 2010 A comparison of plasma and electron beam-sterilization of PU catheters Radiat. Phys. Chem. 79 93-103

[15] Hoffmann M, Bruch H, Kujath P and Limmer S 2010 Cold-plasma coagulation in the treatment of malignant pleural mesothelioma: results of a combined approach Interact Cardio-Vasc Thorac. Surg. 10 502-5

[16] Robert E, Vandamme M, Sobilo J, Sarron V, Riès D, Dozias S, Brulle L, Lerondel S, Le Pape A and Pouvesle J M 2012 First achievements and opportunities for cancer treatment using non thermal plasma Plasma for Bio-Decontamination, Medicine and Food Security (NATO Science for Peace and Security Series A: Chemistry and Biology) ed Z Machala et al (Heidelberg: Springer)

[17] Vandamme M, Robert E, Sobilo J, Sarron V, Riès D, Dozias S, Legrain B, Lerondel S, Le Pape A and Pouvesle J M 2011 In situ application of non-thermal plasma: preliminary investigations for colorectal and lung tolerance Proc. Int. Symp. on Plasma Chemistry-ISPC 20 (Philadelphia, PA)

[18] Xiong Z and Kushner M J 2010 Surface corona-bar discharges for production of pre-ionizing UV light for pulsed high pressure plasmas J. Phys. D: Appl. Phys. 43505204

[19] Rauf S and Kushner M J 1999 Dynamics of a coplanar-electrode plasma display panel cell: I. Basic operation J. Appl. Phys. 85 3460-9

[20] Phelps A V and Petrovic Z Lj 1999 Plasma Sources Sci. Technol. 8 R21-44

[21] Sarron V, Robert E, Dozias S, Vandamme M, Ries D and Pouvesle J M 2011 Splitting and mixing of high-velocity ionization-wave-sustained atmospheric-pressure plasmas generated with a plasma gun Trans. Plasma Sci. 39 2356-7

[22] Xiong Z and Kushner M J 2012 Atmospheric pressure ionization waves propagating through a flexible high aspect ratio capillary channel and impinging upon a target Plasma Sources Sci. Technol. 21034001

[23] Mason N J and Newell W R 1987 J. Phys. B: At. Mol. Phys. 201357

[24] Suzuki T Y, Sakai Y, Min B S, Takayanagi T, Wakiya K, Suzuki H, Inaba T and Takuma H 1991 Phys. Rev. A 435867

[25] Hayashi M 1983 J. Phys. D: Appl. Phys. 16581

[26] Eckstrom D J, Nakano H H, Lorents D C, Rothem T, Betts J A, Lainhart M E, Dakin D A and Maenchen J A 1988 J. Appl. Phys. 641679

[27] Shon J W 1993 PhD Thesis University of Illinois

[28] Teubner P J O, Riley J L, Tonkin M C, Furst J E and Buckman S J 1985 J. Phys. B: At. Mol. Phys. 183641

[29] Rapp D and Englander-Golden P 1965 J. Chem. Phys. 431464

[30] Bretagne J, Godart J and Puech V 1982 J. Phys. D : Appl. Phys. 152205

[31] Shiu Y J and Biondi M A 1978 Phys. Rev. A 17868

[32] Vriens L 1964 Phys. Lett. 8260

[33] Hokazono H, Midorikawa K, Obara M and Fujioka T 1984 J. Appl. Phys. 56680

[34] Ohwa M, Moratz T J and Kushner M J 1989 J. Appl. Phys. 665131 
[35] Levin L A, Moody S E, Klosterman E L, Center R E and Ewing J J 1981 IEEE J. Quantum Electron. 172282

[36] Aymar M and Coulombe M 1978 At. Data Nucl. Data Tables 21537

[37] Kinnari F, Suda A, Obara M and Fujioka T 1983 IEEE J. Quantum Electron. 191587

[38] Holstein T 1947 Phys. Rev. 721212

Holstein T 1951 Phys. Rev. 831159
[39] Xiong Z and Kushner M J 2011 Ionization wave splitting at the T-junction of a dielectric channel Trans. Plasma Sci. $392320-1$

[40] Robert E, Sarron V, Riès D, Dozias S, Vandamme M and Pouvesle J M 2012 Characterization of pulsed atmospheric-pressure plasma streams (PAPS) generated by a plasma gun Plasma Sources Sci. Technol. 21034107 